\title{
STRUCTURE OF CATEGORIES
}

\author{
BY JOHN R. ISBELL
}

Introduction. This paper sets out to develop a structure theory of categories and carries it, not very far, but far enough for some applications. We need a new definition of complete (coinciding with old definitions [2], [8] for well-powered co-well-powered categories). The new definition is needed even to construct images of mappings. With it, we can show that every completion of a small category, and also every primitive category of algebras, is a retract of any category in which it is fully embedded. Such categories are called injective; strictly stronger injectiveness properties are rather trivial. By a completion of $a$ is meant a complete category in which $a$ is fully embedded so that no complete full proper subcategory contains it. The results stated come from the regular completion theory concerning complete extensions of $a$, regularly represented in $\operatorname{Cat}\left(a^{*}, \mathfrak{u}\right)$, and the statements given are simply the main applications of two theorems to the effect that complete categories satisfying certain boundedness conditions are injective. Apparatus is set up, but not developed, for a general completion theory and finer tests for injectiveness.

Precise statements of results cannot well be given before we establish the set-theoretic foundation ( $\$ 1)$. The new clause in the definition of completeness requires every intersection of extremal subobjects [8] to be representable, and the dual. Then every completion of a small category $a$ is well-powered, no intermediate full subcategory is left complete, and the embedding preserves all limits that may exist in a (and dually). Unfortunately, retraction preserves completeness only in the weaker sense of [8]; and, since not every category has a completion in the same Grothendieck universe, and injectiveness is defined relative to a universe, I can prove that an injective category is complete only in the still weaker sense of Freyd [2]. In any of these senses, up to an equivalence of categories, a left complete full subcategory of a complete category is both left closed in its right closure and right closed in its left closure. Accordingly, one would hope, from Freyd's theorems on existence of adjoints, to retract by a reflector and a coreflector. This question is pursued for

Based on address by Professor Isbell delivered before the Athens meeting of the Society on November 20, 1964 by invitation of the Committee to Select Hour Speakers for Southeastern Sectional Meetings; received by the editors October 13, 1965. 
some distance (roughly, the second adjoint functor always exists), but the hypothesis that comes out presents an unsolved problem even in the category of abelian groups.

I am indebted to K. H. Hofmann and P. S. Mostert for patient criticism of many revisions of the first half of this paper.

1. Fundamentals. Constructions in this paper will require something like the Grothendieck theory of universes, but they require less and I take what seems to be a different point of view. The wellknown fact that some basic constructions applied to large categories take us out of the universe seems to me to indicate that the constructions are not yet properly presented. The discovery of proper presentations is too difficult, though, for all work on these constructions to wait for it. Therefore, we assume one Grothendieck universe and, outside it, a collection of improper or extraordinary sets which will be used for improper constructions.

Precisely, we assume a Gödel-Bernays set theory having sets and proper classes and having at least one uncountable strongly inaccessible cardinal number $\infty$, henceforth supposed fixed. We assume for convenience that the class of all sets can be well ordered. We recall that there is a fairly easily defined set $M$ of $\infty$ "hereditarily small" sets which can be regarded as a model for ordinary set theory (a Grothendieck universe; technically, a supercomplete Shepherdson model [13]). A legitimate or "proper" set should be defined as a member of $M$, and a legitimate class or category should be contained in $M$. However, we wish to deemphasize unimportant distinctions between isomorphic or equivalent categories, and therefore we make the following definitions.

A set is small, large, or extraordinary according as its cardinal number is $<\infty, \infty$, or $>\infty$. A category is defined as usual [12] and may be a proper class. A small category is a category whose class of mappings is a small set. A locally small category is one in which every set of coterminal (= coinitial and cofinal) mappings is small. A category is ordinary if it is locally small and has at most $\infty$ objects.

We fix the notation $u$ for the category of all small sets and the functions between them, which is locally small. The category of all sets and functions will be called $\mathcal{U}$ ! A covariant functor $F: \mathfrak{e} \rightarrow \mathcal{U}$ or $F: \mathfrak{C} \rightarrow \mathfrak{U}$ ! will be called a grounding of $\mathfrak{C}$, respectively ordinary or extraordinary. Either modifier might be omitted, but only "extraordinary" will be omitted in this paper, when it is clear from the context. The dual notion $\left(F: \mathfrak{e}^{*} \rightarrow \mathfrak{U}\right.$ or $\left.\mathfrak{U} !\right)$ is cogrounding.

$\mathfrak{e}(X, Y)$ denotes the set of maps from $X$ to $Y$ in $\mathfrak{e}$. The symbol 
$\mathfrak{C}(, X)$, which may be replaced by $h_{X}$ when it is clear what $\mathfrak{e}$ is meant, denotes the cogrounding defined by $h_{X}(W)=\mathfrak{e}(W, X)$, $h_{X}(f)(g)=g f$. The cogroundings $h_{X}$ and their covariant analogues $h^{X}$ are called principal; an unambiguous common name for them is principal functors; and the functors naturally equivalent to them are called representable. If $B$ is a full subcategory of $\mathcal{e}$, the restriction of $\mathcal{C}(, X)$ to $B$ may be designated $B(, X)$, even when $X$ is not in $B$, provided the embedding of $B$ in $\mathcal{C}$ is clear from the context; such a functor is representable in $\mathfrak{C}$.

The categories which, unlike $u$ ! or even $u$, have only a set of mappings, form with their functors a category Cat. Thus Cat(e, D) should mean a set of functors. We enrich the symbol to mean the category of functors (covariant) from $\mathcal{C}$ to $\mathbb{D}$ with their natural transformations; moreover, we use the symbol even if $D$ has a proper class of maps, so long as $\mathfrak{e}$ does not. In this case each functor from $\mathfrak{C}$ to $D$ must take $\mathcal{C}$ into a set-subcategory of $D$, so that by using any of several conventions as to what an element of Cat(e, $D)$ is, it becomes a genuine category.

In particular, for every category $\mathcal{C}$ with a set of objects, the cogroundings of $\mathfrak{e}$ form a category $\operatorname{Cat}\left(\mathfrak{e}^{*}, \mathfrak{u} !\right)$. The correspondence $X \rightarrow h_{X}$ gives a full embedding, the (left) regular representation of $\mathbb{C}$. The right regular representation by groundings is a dual embedding. For ordinary categories we also (perhaps preferably) apply the same terms to the representations by ordinary cogroundings and groundings.

There are also subregular representations $X \rightarrow B(, X)$ (respectively $\Theta(X),) . B$ is left adequate (right adequate) if the (right) subregular representation over $B$ is a full (dual) embedding.

When the name of the category $\mathfrak{C}$ is long or otherwise distracting, we may write $\operatorname{Hom}(X, Y)$ instead of $\mathfrak{e}(X, Y)$. We shall want (mainly for the sequel) to combine the two regular representations in Cat $\left(\mathfrak{e}^{*}, \mathfrak{u}\right.$ !) and Cat(e, $\mathfrak{u}$ !). These functor categories are related by a pair of contravariant functors called conjugations. The conjugate of either a grounding or a cogrounding $F$ is called $F^{*}$ in [6], and this short notation will be convenient; but for clarity we had better define $K_{1}: \operatorname{Cat}\left(\mathfrak{e}^{*}, \mathfrak{u} !\right)^{*} \rightarrow \operatorname{Cat}(\mathfrak{e}, \mathfrak{u} !)$ and $K_{2}: \operatorname{Cat}(\mathfrak{e}, \mathfrak{u} !)^{*} \rightarrow \operatorname{Cat}\left(\mathfrak{e}^{*}, \mathfrak{u} !\right)$. Each $K_{1}(F)=F^{*}: \mathfrak{e} \rightarrow \mathcal{U}$ ! is defined by $F^{*}(X)=\operatorname{Hom}\left(F, h_{X}\right)$, $\left[F^{*}(f)(\Phi)\right]_{W}(p)=f \Phi_{W}(p)$, for $p \in F(W), \Phi \in F^{*}(X), f: X \rightarrow Y$; for $\Psi: F \rightarrow G, K_{1}(\Psi): G^{*} \rightarrow F^{*}$ takes each $\operatorname{Hom}\left(G, h_{X}\right)$ to $\operatorname{Hom}\left(F, h_{X}\right)$ by composition with $\Psi . K_{2}$ is defined dually. The functors $K_{1}, K_{2}$ are adjoint on the right, i.e. the functors of two variables $F$ in $\operatorname{Cat}\left(\mathfrak{e}^{*}, \mathfrak{u}\right.$ !), $G$ in $\operatorname{Cat}(\mathcal{e}, \mathcal{u} !)$, symbolized by $\operatorname{Hom}\left(F, K_{2}(G)\right), \operatorname{Hom}\left(G, K_{1}(F)\right)$, 
are naturally equivalent. The easiest way to see this is by means of a neutral description of the sets $\operatorname{Hom}^{*}(F, G)$ corresponding to

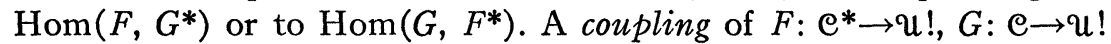
is a function $m$ assigning to every ordered pair $(p, q)$ with $p \in F(X)$, $q \in G(Y)$, a map $m(p, q): X \rightarrow Y$ so that $m(F(f)(p), G(g)(q))=g m(p, q) f$ identically for maps $f$ with range $X$ and $g$ with domain $Y$ in $\mathcal{C}$.

1.1. There is a one-to-one correspondence between couplings $m$ of $F, G$ and natural transformations $\mu: G \rightarrow F^{*}$, defined by

$$
\left[\mu_{Y}(q)\right]_{X}(p)=m(p, q) .
$$

We omit the routine verification. Combining 1.1 with its dual and a verification of naturality, one gets the adjointness of $K_{1}$ and $K_{2}$, which we will not use explicitly.

A grounding couple consists of a cogrounding $F$, a grounding $G$, and a coupling $m$. The couple category $\mathrm{Co}(\mathfrak{e}, \mathfrak{u}$ !) has these couples for objects, and for mappings the conjoint transformations $\left(F_{1}, G_{1}, m_{1}\right)$ $\rightarrow\left(F_{2}, G_{2}, m_{2}\right)$, which are pairs of natural transformations $\Phi: F_{1} \rightarrow F_{2}$, $\Psi: G_{2} \rightarrow G_{1}$, satisfying $m_{2}\left(\Phi_{X}(p), q\right)=m_{1}\left(p, \Psi_{Y}(q)\right)$. There is a full embedding of $\mathfrak{e}$ in $\operatorname{Co}(\mathcal{C}, \mathcal{U} !)$ taking each $X$ to the principal couple $\left(h_{X}, h^{\mathbf{x}}, \cdot\right)$; we call this the double regular envelope of $\mathfrak{e}$. Both regular representations are factors of the double regular envelope in a natural

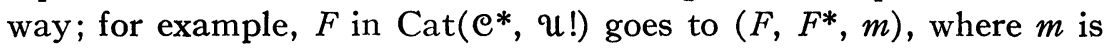
the coupling corresponding by 1.1 to $1: F^{*} \rightarrow F^{*}$. These functors are again full embeddings.

$\mathrm{Co}(\mathfrak{e}, \mathfrak{U} !)$ is a high first approximation to a largest reasonable extension of $\mathfrak{e}$. To adjoin any object $X$ to $\mathfrak{e}$ we need the coupled functors $\mathfrak{e}(, X)$ and $\mathfrak{e}(X$,$) . In requiring that the adjoined objects$ map just by conjoint transformations we are, of course, imposing a restriction.

We note a second approximation to a largest reasonable extension. A grounding couple $(F, G, m)$, or the coupling $m$, is separated if whenever $p \neq p^{\prime}$ in $F(X)$, there exist $Y$ and $q$ in $G(Y)$ such that $m(p, q)$ $\neq m\left(p^{\prime}, q\right)$, and dually.

1.2. Couplings $m$ of principal functors $h_{X}, h^{Y}$ are determined by the map $m\left(1_{X}, 1_{Y}\right)=m^{1}$ and are separated if and only if $m^{1}$ is bimorphic (epimorphic and monomorphic).

The proof is a routine application of the Yoneda lemma which says that the regular representations are full embeddings and consequently $h_{X}, h^{X}$ are conjugate.

REMARK. For sets, (suitable) spaces with base point, abelian groups and Banach spaces, at least, there is a Fuks-Švarc duality $D: \operatorname{Cat}(\mathcal{e}, \mathfrak{e})^{*} \rightarrow \operatorname{Cat}(\mathcal{C}, \mathcal{C})$, self-adjoint on the right. (See [12] for 
references, under Fuks, Švarc, Mitjagin, and combinations.) One naturally wants to compare with conjugations $K_{1}, K_{2}$. These categories have extensions $\varepsilon$ in which $D$ turns in to a categorical duality $D_{1}: \mathcal{E}^{*} \leftrightarrow \mathcal{E}$ and there is a second-order duality $D_{2}$ for functors. Using $D_{1}$, one can derive $K_{1}$ and $K_{2}$ from a duality $K$ of the same nature as $D_{2}$ (contravariant on $\operatorname{Cat}(\mathcal{E}, \mathcal{E})$ to itself). $K$ and $D_{2}$, though similarly constructed, do not commute.

2. Limits and factorization. A contravariant ideal of a category $\mathfrak{C}$, in an object $X$, is an arbitrary subfunctor $I$ of $\mathfrak{e}(, X)$. (That is, $I(W) \subset \mathfrak{e}(W, X)$ and maps by restrictions.) The representable contravariant ideals are [8] the principal ideals generated by monomorphisms $m: S \rightarrow X$; these will be called subobjects in the wide sense, or wide or mono subobjects. (They are in a natural one-to-one correspondence with the subobjects of Grothendieck [4], Freyd [2]. The partial ordering [4] reduces in the present formulation to the subfunctor ordering.)

In suitable contexts we may refer to a monomorphic generator $m$ or even to an object $S$ as a "subobject" of $X$. Less drastically, any ideal $I \subset h_{X}$ may be confused with the union of its values $I(W)$. (This is a "right ideal" in Eckmann-Hilton [1].) In the same spirit, a functor $F: \mathbb{e} \rightarrow \mathcal{~ ! ~ i s ~ s a i d ~ t o ~ b e ~ g e n e r a t e d ~ b y ~ a ~ s e t ~ o f ~ v a l u e s ~ o f ~} F$ or elements of values of $F$ if no proper subfunctor has all those values, respectively elements of values. $F$ is properly generated if it is generated by some small set of elements of its values.

Generators suggest relations. An ideal $I$ in $X$ is called strict if there is a set of pairs of maps $h_{\alpha}: X \rightarrow Y_{\alpha}, k_{\alpha}: X \rightarrow Y_{\alpha}$, such that each value $I(W)$ is the set of all $f: W \rightarrow X$ satisfying $h_{\alpha} f=k_{\alpha} f$ for all $\alpha$. If $I$ is at the same time a wide subobject, then it is called a strict subobject. A monomorphism is strict if the subobject it generates is strict.

Strict ideals are a special case of limit functors, and their representations (strict subobjects) are limits. In general, a diagram $D$ in a category $\mathcal{C}$ is a functor $D: D \rightarrow \mathcal{C}$ whose domain category is a set. ${ }^{1}$ The limit functor $L_{D}$ of $D$ is defined by means of the functor $K: \mathfrak{C}$ $\rightarrow \operatorname{Cat}(D, \mathcal{C})$ which takes each object $W$ to the constant functor $K_{W}$ all of whose values are $1_{W} ; L_{D}$ is $h_{D} K$. A limit of $D$ is a representation of $L_{D}$. The dual concept is colimit.

Diagrams, at least the finite ones, can be drawn; a mapping (or object=identity mapping) "in" $D: D \rightarrow C$ is a mapping $g: X \rightarrow Y$ of $\mathcal{C}$ indexed by a mapping $f$ of $D$ such that $D(f)=g$. Maps $\Phi: K_{W} \rightarrow D$ correspond to left conical extensions (1.c.e.'s) of $D$ in which $W$ is

1 Possibly extraordinary. 
attached to each object $X$ in $D$ by one map, so that all the triangles

$$
\begin{array}{r}
W \rightarrow X \\
\searrow \quad \downarrow \\
Y
\end{array}
$$

are commutative. A limit corresponds to a universal 1.c.e. $W_{0} \rightarrow \cdots$, from which each other 1.c.e. arises by multiplying in a unique map $W \rightarrow W_{0}$. The picture helps in seeing some properties of limits, e.g. the following. If a diagram $D$ is the union of a directed set of subdiagrams $D_{\alpha}$ having limits $L_{\alpha}$, then the inverse mapping system $D^{\prime}$ formed by the $L_{\alpha}$ and the obvious bonding maps (a conical extension is conical over any subdiagram) has the same limit functor as $D$. This is a rule for expressing limits as limits of limits, and there is another such rule: a limit of a diagram $D$ is a strict subobject of a direct product ${ }^{2}$ of all the objects in $D$, with the defining relations $h_{\alpha} f=k_{\alpha} f$ expressing commutativity of the triangles (2.0). Unfortunately, there is no rule for expressing limits of limits (of diagrams in a subcategory $B$ ) as simple limits (of diagrams in $\beta$ ), and it is not generally possible.

The formula going (in one direction) from a limit $\Psi: h_{X} \rightarrow h_{D} K$ to a universal 1.c.e. $\Phi$ is $\Phi=\Psi_{X}\left(1_{X}\right)$. The inverse formula would require more notation. A limit functor $L_{D}: \mathfrak{C}^{*} \rightarrow \mathfrak{U}$ ! is a limit object of the diagram in $\operatorname{Cat}\left(\mathfrak{e}^{*}, \mathfrak{u} !\right)$ consisting of $D$ followed by left regular representation. The colimit functor $R_{D}$ is a limit object of a similar diagram. The colimits in the functor categories are less interesting in themselves, but they show the following.

2.1. The limit functors on a category with a set of objects are precisely the conjugates of groundings.

Proof. Every limit is a conjugate. For, given $D$, define $R_{D}^{0}: \mathcal{e} \rightarrow \mathcal{U}$ ! as follows. Each value $R_{D}^{0}(Y)$ is a disjointed union of the sets $h^{D(I)}(Y)$, as $I$ ranges over the objects of $D$, reduced by identification of all pairs $f: D(J) \rightarrow Y, f D(e): D(I) \rightarrow Y$. Note that the notation $f: D(J) \rightarrow Y$ must be read carefully; $J$ matters as well as $D(J)$. For a map $p \in \mathfrak{e}(Y, Z), R_{D}^{0}(p)$ takes the equivalence class of $f: D(J) \rightarrow Y$ to the equivalence class of $p f$. Consider the values of the conjugate functor, $R_{D}^{0 *}(W)=\operatorname{Hom}\left(R_{D}^{0}, h^{W}\right)$. Given an element $\Phi: R_{D}^{0} \rightarrow h^{W}$, define for each object $I$ of $D$ the map $\Phi^{\sharp}(I) \in h^{W} D(I)$ which is the value of $\Phi_{D(I)}$ on the equivalence class of the identity $1: D(I) \rightarrow D(I)$. By routine checking, the values $\Phi^{\#}(I)$ determine $\Phi$ and naturality of $\Phi$ is equivalent to conicality of $\Phi^{\#}$. Finally, since both $L_{D}$ and $R_{D}^{0 *}$ take maps $a: V \rightarrow W$ to multiplication by $a$, we have a natural equivalence.

2 "Direct" for emphasis. The dual is coproduct. 
To conclude, limits are the same as conjugates of functors $R_{D}^{0}$. If $\mathcal{C}$ has a set of objects, it is a simple exercise to verify that every $G: \mathfrak{e} \rightarrow \mathcal{U}$ ! is an $R_{D}^{0}$, proving 2.1 .

Inspection shows that $R_{D}^{0}$ is a colimit object of the diagram in Cat(e, $u$ !) which is the composition of $D$ with the right regular representation of $\mathfrak{e}$. Thus every grounding is a colimit of principal groundings. We shall need to apply a variant of this result:

2.1.a. Every ordinary cogrounding on a small category is a colimit of a small diagram in the principal cogroundings.

It may be noted that the concept of limit was introduced by Kan [10] in 1958, the equivalent concept of conjugate by me [6] in 1960, and the equivalence was apparently unnoticed till now. In spite of the equivalence, both concepts seem to be justified.

Every intersection of strict ideals in $X$ is a strict ideal; but an intersection of wide (or strict) subobjects need not be a wide subobject. For any map $f: W \rightarrow X$, the smallest strict contravariant ideal in $X$ including $f$ is the dominion of $f$. If the intersection of all wide subobjects of $X$ which include $f$ is representable, it is the left image of $f$. The dual terms are codominion and right image. Since there are so many of them, and since the proposed terminology is all new, let us look at the "normal" relations of these objects before inquiring $(2.4,2.7)$ how well-behaved a category must be for the relations to be normal. The normal relations hold in particular in every equationally definable (primitive) category of algebras.

$$
W \rightarrow C \rightarrow L \rightarrow R \rightarrow D \rightarrow X .
$$

The domain $W$ maps by a strict epimorphism $q$ to the codominion $C$; every strict epimorphism that is an initial factor of $f$ is an initial factor of $q$. The maps $C \rightarrow L$ and $W \rightarrow L$ are epimorphic, and so are all the maps in the figure with range $R$. The dual statements are true too so, in particular, $L \rightarrow R$ is bimorphic. Briefly, the peculiarity is that although $L$ and $D$ are constructed similarly by intersecting subobjects of $X$, and $C$ and $R$ are constructed starting from $W, L$ comes out closer to $W$.

Grothendieck [4] and Freyd [2] call $L$ the image of $f$ and $R$ the coimage. This seems to me mildly confusing on this level, and worse in some examples.

The rest of this section concerns conditions for "normal" behavior of images. A monomorphism $i$, and the subobject it generates, are called extremal if every epimorphic initial factor $k$ of $i(i=j k)$ is an isomorphism; and dually.

Suppose that $f: W \rightarrow X$ has both a left image $m: L \rightarrow X$ and a right image $e: W \rightarrow R$. We have 


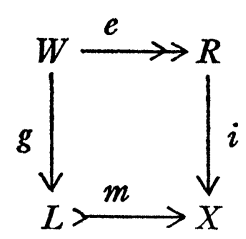

2.2. If $i$ is monomorphic then there is a unique and bimorphic map $t: L \rightarrow R$ preserving commutativity. If every map has a right image mapping monomorphically into the range, then every left image (that may exist) is an extremal quotient of the domain, and every right image is an extremal subobject of the range; and dually.

PRoof. If $i$ is monomorphic then the intersection $m$ is a multiple of it, $m=i t$. Here $t$ is unique because $i$ is monomorphic; $t$ is monomorphic because $i t$ is monomorphic: $t g=e$ because $i t g=i e ; t$ is epimorphic because $t g$ is epimorphic. If every map has a factorization (e.g. over a right image) monomorphism-epimorphism, then $g$ does; since $m$ generates the smallest subobject of $X$ including $f$, the monomorphic factor of $g$ must be isomorphic, and $g$ is an extremal epimorphism. Similarly, since $e$ generates the smallest quotient of $W$ including $f$, the monomorphism $i$ must be extremal.

Freyd [2] calls a category "left complete," and we shall call it left small-complete, if every small diagram (i.e. with small domain) has a limit. We need not one but three somewhat stronger completeness conditions, the strongest of which is that, besides left small-completeness, every intersection of wide subobjects of an object is a wide subobject: left wide-completeness. Since intersections are limits, these notions and the intermediate ones coincide in the important case of well-powered categories, i.e. those in which each object has only a small set of wide subobjects. It should be noted, too, that although a rich variety of examples illustrate the degrees of completeness and the necessity for strong completeness assumptions in some of the theorems, all known examples seem artificial. ${ }^{3}$

A category is left complete (left extremal-complete for emphasis) if it is left small-complete and every intersection of extremal subobjects of an object is representable. Fortunately this implies $\left(2.4\right.$ below $\left.^{4}\right)$ that these intersections are again extremal subobjects. Finally, a left

\footnotetext{
${ }^{3}$ Even the distinction between left and right completeness is not easy to illustrate with everyday examples. Gaifman [3] and Hales [5] have shown that the left widecomplete category of all small complete Boolean algebras and complete homomorphisms is not right small-complete. The related category of all topological spaces and continuous open mappings is right extremal-complete but may not be right widecomplete.

${ }^{4}$ Assuming the category has only a set of objects.
} 
small-complete category is left strict-complete if every strict contravariant ideal is representable. (Since those defined by small sets of relations would be representable anyway, this is still an intersection condition.)

The strengths of the four notions are "wide" $\Rightarrow$ "extremal" $\Rightarrow$ "strict" $\Rightarrow$ "small." This is obvious, using the known lemma [8] that every strict monomorphism is extremal. There is a sufficient condition for small-completeness (left or right) to imply strict-completeness that is weak and hereditary: embeddability in $u$ (stated in [8] for ordinary categories, but the proof [7] generalizes at once).

Instead of considering limits of certain diagrams one could look at conjugates of certain functors. We omit details, remarking that it is easy to find manageable characterizations of left small-completeness and left strict-completeness in these terms, but not the others.

Obviously, the existence of left or right images follows directly from left or right wide-completeness. It is known that one can prove existence and fairly normal behavior by approaching right images from the right (i.e. from the range; unlike the definition). We can strengthen the known theorem [8] by taking more pains with the proof. The somewhat elaborate machinery is going to be used more than once.

A left multistrict analysis is an inverse mapping system $\left\{X_{\alpha} ; f_{\beta \alpha}\right\}$ indexed by an initial set of ordinal numbers, in which each bonding mapping $f_{\alpha+1, \alpha}$ is a strict monomorphism and each $X_{\beta}$ indexed by a limit number is the inverse limit of its predecessors, with coordinate projections $f_{\beta \alpha}$. The inverse limit condition is equivalent to the condition that $f_{\beta 0}$ is an intersection of the preceding monomorphisms $f_{\alpha 0}$. A multistrict monomorphism is a mapping appearing in some left multistrict analysis.

2.3. Every multistrict monomorphism is an extremal monomorphism.

Proof. We may suppose the given mapping occurs as $f_{\alpha 0}$ in a left multistrict analysis $\left\{X_{\alpha} ; f_{\beta \alpha}\right\}$. By remarks above, it is a monomorphism. Suppose $f_{\alpha 0}=g e$, where $e$ is epimorphic. We prove inductively that for each $\beta$ there exists $g_{\beta}$ such that $f_{\beta 0} g_{\beta}=g$. Note that this holds for $\beta=0$; that $g_{\beta}$ will be unique, since $f_{\beta 0}$ is monomorphic; and that $g_{\beta} e$ will be $f_{\alpha \beta}$, since $f_{\beta 0} g_{\beta} e=g e=f_{\alpha 0}=f_{\beta 0} f_{\alpha \beta}$. To get from $\beta$ to $\beta+1$, every equation $h f_{\beta+1, \beta}=k f_{\beta+1, \beta}$ implies $h g_{\beta} e=k g_{\beta} e ; h g_{\beta}=k g_{\beta}$; therefore $g_{\beta}$ is a multiple $f_{\beta+1, \beta} g_{\beta+1}$ of the strict monomorphism $f_{\beta+1, \beta}$. At a limit ordinal $\gamma$, the preceding $g_{\beta}$ are the coordinates of the required mapping $g_{\gamma}$, and the induction runs. Finally, since $f_{\alpha 0} g_{\alpha} e=g e=f_{\alpha 0}, g_{\alpha} e$ is an identity. As $e$ is epimorphic, it is isomorphic, which was to be shown.

2.4. THEOREM. In a left complete category which has only a set of objects, every mapping has a right image, which maps monomorphically 
into the range; every extremal monomorphism is multistrict, so that compositions of extremal monomorphisms are extremal; and intersections of extremal subobjects are extremal subobjects. The right image of a mapping is the smallest extremal subobject that includes it.

Proof. Given $f: X \rightarrow Y$, we construct a multistrict analysis inductively, factoring $f=m_{\beta 0} f_{\beta}$ across $m_{\beta 0}: Y_{\beta} \rightarrow Y$. Each bonding mapping $m_{\beta+1, \beta}$ is chosen as a monomorphic generator of the dominion of $f_{\beta}$. In this construction, objects $Y_{\beta}$ may be repeated, but the maps $m_{\beta 0}$ are all different, until $m_{\alpha+1, \alpha}$ is an isomorphism. This can happen only when $f_{\alpha}$ is epimorphic. For any factorization $f=g e$, where $e$ is epimorphic, $g$ factors through every $m_{\beta 0}$, by a routine induction. Then $e$ is a factor of $f_{\alpha} ; f_{\alpha}: X \rightarrow Y_{\alpha}$ is a right image of $f$, and $Y_{\alpha}$ is a monosubobject (and extremal, by 2.2) of the range.

In case $f$ was an extremal monomorphism, the epimorphic factor $f_{\alpha}$ is an isomorphism and $f$ is multistrict. For a composition, juxtapose analyses.

For an intersection of subobjects of $X$ generated by extremal monomorphisms $m_{\alpha}$, there is (by left completeness) a monomorphic generator $n$. Factor $n=p e$ across its right image $e$. We have also factorizations $n=m_{\alpha} u_{\alpha}$; and by the same sort of induction as in 2.3, $p=m_{\alpha} v_{\alpha}$. Hence $p$ is a lower bound for all $m_{\alpha}$ and must factor across $n, p=n f=p e f$. Then $e f$ is an identity. Since $e$ is a right factor of $n$, it is monomorphic; as a monomorphic left factor of an identity, it is isomorphic, and $n$ is extremal.

For the final assertion, it is obvious from the construction that the right image is the smallest including multistrict subobject. We remark that this assertion can be proved without multistrict analyses whenever, besides the hypotheses in 2.2 , one knows that compositions of extremal monomorphisms are extremal.

2.5. COROLlaRY. In a left wide-complete category having only a set of objects, every mapping $f$ factors across its left and right images, $f=a b c$, where $c$ is an extremal epimorphism, $b$ a bimorphism, and a an extremal monomorphism.

The right image comes from 2.4 , the left image directly by intersecting, and the rest from 2.2. The factorization theorem in [8] gives the information in 2.4 and 2.5 for well-powered categories. It is not known even in that case whether extremal epimorphisms are multistrict, or compose, or intersect.

2.6. REMARK. Of course 2.4 and 2.5 do not require completeness. The limits involved are subobjects; the properties used might be called "completeness downward." The next results 2.7 and 2.8 are implied by completeness downward, and 2.9 can be adapted, trivially, 
to a criterion for completeness downward. Similar remarks apply, without difficulty, to several later results.

2.7. TheOREM. In a complete category having only a set of objects, the factorization (2.7) is functorial.

Proof. The objects of the domain category of the factorizing functor $S$ are short diagrams $f: W \rightarrow X$ in $\mathcal{e}$, or for short, $f$. Maps $\alpha: f \rightarrow f^{\prime}$ are natural transformations of diagrams, which amount to pairs of maps $\alpha_{1}: W \rightarrow W^{\prime}, \alpha_{2}: X \rightarrow X^{\prime}$, with $f^{\prime} \alpha_{1}=\alpha_{2} f . S(f)$ is a diagram (2.7), unique up to an equivalence of diagrams inducing the identity on the subdiagram $W \rightarrow X$. We wish to define $S(\alpha): S(f) \rightarrow S\left(f^{\prime}\right)$ so as to induce $\alpha$ on the subdiagram; this means precisely to construct

$$
\begin{aligned}
W \rightarrow C \rightarrow & L \rightarrow R \rightarrow D \stackrel{m}{\rightarrow} X \\
\downarrow & \downarrow \quad \downarrow \quad \downarrow \quad \downarrow \quad \downarrow \quad \downarrow \quad \alpha_{2} \\
W^{\prime} \rightarrow C^{\prime} \rightarrow L^{\prime} \rightarrow R^{\prime} \rightarrow & D^{\prime} \rightarrow X^{\prime},
\end{aligned}
$$

commutative, with the boundary of the picture consisting of the data $S(f), S\left(f^{\prime}\right), \alpha$. Consider the strict subobject of $X^{\prime}$ generated by $D^{\prime} \rightarrow X^{\prime}$, which is defined by some family of equations $h_{\lambda} g=k_{\lambda} g$. We have, for all $\lambda, h_{\lambda} f^{\prime}=k_{\lambda} f^{\prime}$, whence $h_{\lambda} f^{\prime} \alpha_{1}=k_{\lambda} f^{\prime} \alpha_{1}, h_{\lambda} \alpha_{2} f=k_{\lambda} \alpha_{2} f$. Since $m$ generates the smallest strict subobject of $X$ including $f, h_{\lambda} \alpha_{2} m$ $=k_{\lambda} \alpha_{2} m$, and $\alpha_{2} m$ factors across $D^{\prime}$. The rectangle $D X^{\prime}$ is commutative by construction; the rectangle $W D^{\prime}$ is commutative since $D^{\prime} \rightarrow X^{\prime}$ is monomorphic. Now insert the dominions $D_{2}$ of $W \rightarrow D$, $D_{2}^{\prime}$ of $W^{\prime} \rightarrow D^{\prime}$. By the same proof, there is a connecting map $D_{2} \rightarrow D_{2}^{\prime}$. By an obvious transfinite induction, one arrives finally at $R \rightarrow R^{\prime}$. Duality yields $C \rightarrow C^{\prime}$ and $L \rightarrow L^{\prime}$. Finally, $S$ is functorial because the placement of epi- and monomorphisms assures that the diagram-filling problem has only one solution.

If one specializes the diagram in the foregoing proof by assuming isomorphisms in several places, one gets a number of corollaries, most of which are uninteresting or evident. There is a curious exception.

2.8. Corollary. In a left strict-complete category, if $f: W \rightarrow X$ is an initial factor of a strict monomorphism, then $W$ is a retract of the dominion of $f$.

Proof. Left strict-completeness yields dominions and the map connecting them in 2.7. Then if $W \rightarrow W^{\prime} \rightarrow D^{\prime}$ is an isomorphism, $W \rightarrow D$ has a left inverse.

The two-step multistrict monomorphism $f$ need not be strict, even in the category of semigroups (5.1). 
For each of the four degrees of (left) completeness, there is a test somewhat more convenient than the definition. For left small-completeness, it is simply that every small family of objects has a product and every coterminal pair of maps an equalizer ( $=$ limit, for a coterminal pair) [2]. For left strict-completeness, every small family has a product and every strict contravariant ideal is representable. For left wide-completeness, I am not sure the test can be useful, but it seems likely; it is simply that instead of intersecting a structureless family of subobjects $\left\{m_{\alpha}: \alpha \in A\right\}$, one can well-order $A$ and use the descending chain of partial intersections $\cap\left\{m_{\alpha}: \alpha<\beta\right\}$. Finally:

2.9. A left small-complete category having only a set of objects is left complete if (and only if) every left multistrict analysis has a limit.

The routine, but tedious, proof will be sketched. All strict contravariant ideals are representable, by a multistrict analysis along initial segments of a well-ordered set of defining relations. This justifies the beginning of the proof of 2.4. Then, noting that small intersections are representable, and proving along the way that intersections are (1) extremal and (2) multistrict, one gets every intersection of extremal subobjects from a multistrict analysis.

The "set of objects" condition in 2.4-2.9 is, of course, no hindrance to using the results for any legitimate purpose. However, we have illegitimate purposes in mind, in functor categories (for example). Note, therefore, that it suffices to assume that each object has only a set of wide subobjects; in that case we call the category set-powered. Its subcategories are another matter.

3. Retracts and adjoints. Though functor categories will occur in this section, all categories not named as functor categories are assumed to have only a set of objects. This will cost something later, when we must refer back to proofs; but not much.

3.1. ThEOREM. If $\&$ is a subcategory and a retract of $\mathfrak{C}$, then every diagram in $\mathrm{B}$ having a limit in $\mathrm{C}$ has a limit in $\mathrm{B}$.

REMARK. We cannot simply say "retractions preserve limits" (5.2). One might say "retractions cherish limits."

Proof. Let $\alpha: \mathfrak{C} \rightarrow ß$ be a retraction. Observe that every idempotent map $k: X \rightarrow X$ in $B$ splits, into factors $r: X \rightarrow R, i: R \rightarrow X$, such that $i r=k, r i=1_{R}$. For $k$ splits in $\mathcal{e}$, in to $\rho: X \rightarrow P, \iota: P \rightarrow X$. (To prove this let $\iota$ be an equalizer of $k$ and $1_{x}$ and factor $k$ across it.) Then if $r=\alpha(\rho), i=\alpha(\iota), i r$ is $\alpha(\iota \rho)=k$ and $r i$ is $\alpha(\rho \iota)$, an identity.

Now, given a diagram $D: D \rightarrow B$ having a limit $\left(X, \Phi: K_{X} \rightarrow D\right)$ in $\mathfrak{C}$, we shall show that $D$ has a limit in $\beta$. Let $\alpha(X)=Y$. We have maps (for each object $A$ of $D$ ) $\alpha\left(\Phi_{A}\right)=\prod_{A}: Y \rightarrow D(A)$ in $B$. Since $\alpha$ is a 
functor, $\Pi$ is a natural transformation from $K_{Y}$ to $D$ and is induced by a unique mapping $\pi: Y \rightarrow X$ in e. Let $\alpha(\pi)=p: Y \rightarrow Y$. For every $\operatorname{map} \tau: T \rightarrow X$ in $\mathcal{e}$ such that all $\Phi_{A} \tau$ are in $ß, \Phi_{A} \tau=\alpha\left(\phi_{A}\right) \alpha(\tau)=\prod_{A} \alpha(\tau)$ $=\Phi_{A} \pi \alpha(\tau)$ for all $A$; thus $\pi \alpha(\tau)$ induces the same transformation $\left(K_{T} \rightarrow D\right)$ as $\tau, \pi \alpha(\tau)=\tau$. Hence $p \alpha(\tau)=\alpha(\pi \alpha(\tau))=\alpha(\tau)$. In particular, $p^{2}=p$.

Consequently, $p$ splits as ir in $B$, where $r i$ is an identity $1_{U}$. We claim that the natural transformation from $K_{U}$ to $D$ defined by the mappings $\prod_{A} i$ is a limit of $D$ (in $B$ ). Considering the limit $\Phi$, we need only show that given $\tau: T \rightarrow X$ such that all $\Phi_{A} \tau$ are in $B$, there is a unique $t: T \rightarrow U$ in $B$ such that $\pi i t=\tau$. One such $t$ is $r \alpha(\tau)$; for $\pi i r \alpha(\tau)=\pi p \alpha(\tau)=\pi \alpha(\tau)=\tau$. Moreover, if $t^{\prime} \neq t$ in $B, \pi i t^{\prime} \neq \pi i t$ since $\alpha(\pi i)=p i=i r i=i$, a monomorphism. This completes the proof.

It follows, of course, that retracts of strict-complete categories are strict-complete. One cannot substitute "complete" or "wide-complete" $(5.3,5.4)$.

For constructing retractions one naturally looks first to the adjoint functor theorems. We take the notions of adjoint and coadjoint as known [12]; but we reformulate the definition, to clarify the generalization coming up. Given $F: \mathfrak{C} \rightarrow \mathbb{D}$, we consider the left regular representations $L_{\Gamma}: \Gamma \rightarrow \operatorname{Cat}\left(\Gamma^{*}, \mathcal{U} !\right)$ for $\Gamma=\mathcal{C}, D$, and the functor $L(F)$ ("Cat $\left(F^{*}, \mathcal{U} !\right)$ ") from cogroundings $J$ of $D$ to cogroundings $J F$ of $\mathrm{e}$. We call a diagram of categories and functors type-commutative if every two functors $P: \Gamma \rightarrow \Delta, Q: \Gamma \rightarrow \Delta$ obtained by following the arrows are naturally equivalent. Then $G: D \rightarrow \mathcal{C}$ is coadjoint to $F$ if

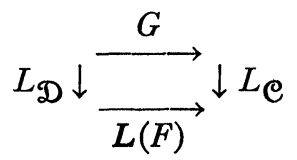

is type-commutative. That is, up to a natural equivalence, $L(F) L_{\mathscr{D}}$ goes into principal functors. It is obvious that this happens if and only if $L(F) L_{\mathscr{D}}$ goes into representable functors (each $h_{X} F: \mathfrak{C} \rightarrow \mathcal{U}$ ! is representable); and it is known ${ }^{5}$ that this is equivalent to the twovariable definitions; $\mathfrak{D}(F(), \quad)$ and $\mathfrak{e}(, G())$ are naturally equivalent. We shall exploit an advantage of that definition by sometimes referring to the adjoint $F$ as the left adjoint, or to $G$ as the right adjoint. ${ }^{6}$

5 [12, Proposition 8.3]. One might as well deduce this from the isomorphism between $\operatorname{Cat}\left(\mathscr{D}, \operatorname{Cat}\left(\mathfrak{C}^{*}, \mathcal{U} !\right)\right)$ and $\operatorname{Cat}\left(D \times \mathfrak{C}^{*}, \mathcal{U} !\right)$.

- Another useful mnemonic: $F$ and $G$ are adjoint in that order if $G$ is the standard Grounding of a category of sets with structure and $F$ takes sets to the Free objects they generate. 
Of course, one actually finds (right) adjoints locally, by showing that each $h_{Y} F$ is representable. We need to justify the method not only for adjoints but for partial adjoints. A pre-coadjoint $G$ of $F: \mathfrak{C} \rightarrow D$ is a functor defined on the full subcategory $Q$ of $D$ whose objects are exactly those $X$ for which $h_{X} F$ is representable, taking it into $\mathbb{e}$, such that $L_{\mathfrak{C}} G$ and $L(F) L_{D} \mid Q$ are naturally equivalent.

We could prove the existence of pre-coadjoints (the first onetwentieth of Theorem 3.9) at once. As the reference "3.9" suggests, there are several other things before that. Many of them concern definitions.

We call a (partial) functor $G: a \rightarrow \mathfrak{C}$, where $Q$ is fully embedded in $D$ by $I: Q \rightarrow D$, left conservative in $D$ if for every diagram $E: \mathcal{E} \rightarrow Q$ such that $I E$ has a limit $\left(X, \Phi: K_{X} \rightarrow I E\right)$, (1) if $G E$ has a limit, then $X$ is in $I(Q)$, and (2) if $X$ is in $I(Q)$, so that $\Phi$ comes from $\Psi: K_{A} \rightarrow E$, then the induced left conical extension $\operatorname{Cat}(\mathcal{E}, G)(\Psi): K_{G(A)} \rightarrow G E$ is universal. $G$ is left conservative if it is left conservative in its domain, i.e. it preserves all limits. (The relative notion may be paraphrased: $I$ and $G$ together reflect limits, and $G$ preserves whatever limits $I$ preserves.) $G$ is left small-closed if its domain is left small-complete and $G$ preserves limits of small diagrams; and left strict-closed, left closed, left wide-closed have obvious parallel definitions.

If $\mathbb{B}$ is a subcategory of $\mathcal{C}$ and the embedding functor is left smallclosed, we call $B$ an essentially left small-closed subcategory; for $B$ to be a left small-closed subcategory we require further that every isomorphism in $\mathbb{C}$ whose domain is in $B$ is in $B$. This is a little tricky. The left small-closed subcategories are well-behaved, being those subcategories $B$ such that every limit of a small diagram in $B$ exists and belongs to $B$. Thus they are closed under intersection, and one could define a left small-closure. But one should not use the notion without realizing that the adjunction of isomorphisms can destroy the structure of a subcategory.

For full subcategories there is no such difficulty. Two subcategories embedded by $I: \mathfrak{Q} \rightarrow \mathfrak{C}, J: ß \rightarrow \mathcal{C}$ are equivalent in $\mathcal{C}$ if there exist functors $S: Q \rightarrow B, T: \beta \rightarrow Q$ such that $S T$ and $T S$ are naturally equivalent to identities, $J S$ naturally equivalent to $I$, and (therefore) $I T$ naturally equivalent to $J$.

3.2. Every essentially left small-closed full subcategory of a category $\mathfrak{C}$ is equivalent in $\mathfrak{e}$ to a unique left small-closed subcategory, which is full.

The proof is easy and is omitted. Moreover, with the parallel definitions for (essentially) left strict-, or (extremal-), or wide-closed subcategories, the results parallel to 3.2 are equally easy.

As an intersection of full subcategories is full, there are full closure operators. But two remarks are needed. As to wide-closure, while 
monomorphisms depend on the category, the notion is absolute for essentially left small-closed subcategories; for $f: X \rightarrow Y$ is monomorphic if and only if the left conical extension of

$$
X \stackrel{f}{\rightarrow} Y \stackrel{f}{\leftarrow} X
$$

by $X$ (with $1_{x}, f, 1_{x}$ ) is universal. As to extremal-closure, one can use 2.9; but there is more to be said.

Even a full left small-closure cannot be formed by simply adjoining limits (once). If $a$ is a full subcategory of $\mathfrak{C}$, we define an $\mathfrak{Q}$-sesquistrict monomorphism $f: X \rightarrow Y$ as a strict monomorphism representing a subobject defined by a set of relations $h_{\alpha} f=k_{\alpha} f$, where $h_{\alpha}$ and $k_{\alpha}$ are coterminal maps with domain $Y$ and with range $A_{\alpha}$ in $Q$. An Q-multistrict monomorphism is a multistrict monomorphism occurring in a left multistrict analysis all of whose immediate bonding maps $f_{\alpha+1, \alpha}$ are $a$-sesquistrict.

3.3. The full left closure of a full subcategory a of $\mathfrak{e}$ (assuming $a$ lies in some left closed full subcategory) has for objects the Q-multistrict subobjects of small products of objects of a.

The only nontrivial point of the proof is in verifying closure under products, and that is not hard.

3.3.a. Parallel results hold, by parallel proofs, for small- and strictclosure. For the strict-closure, the multistrict analyses must have small length; for the small-closure, further, the bonding sesquistrict monomorphisms must have small sets of defining relations.

Describing the left wide-closure is harder, and we will not need it.

Extremal-closure is closely related to the notion of generating. An object $X$ of a category $\mathcal{C}$ is called a generator if for every monomorphism $m: Y \rightarrow Z$ that is not an isomorphism, there exists a map $X \rightarrow Z$ not (left) divisible by $m$. Restating, not all of $\operatorname{Hom}(X, Z)$ is divisible by any such $m$. Given suitable completeness, there is a "universal" map $s: \Sigma \rightarrow Z$, where $\Sigma$ is a coproduct of copies of $X$ indexed and mapped by the elements of $\operatorname{Hom}(X, Z)$; and generating means that $s$ is an extremal epimorphism. In general, a set $S$ of objects of $\mathbb{C}$, or a subcategory $\mathcal{S}$ on the objects of $S$, generates $\mathcal{e}$ if for every $Z$ in $\mathfrak{e}$ there is a small subset $R$ of $S$ such that not all maps from objects of $R$ to $Z$ are at once divisible by a monomorphism $m: Y \rightarrow Z$ that is not an isomorphism. (The requirement that $R$ be small is an innovation, I think, but large generating sets have been little used. We need $R$ small for the next proposition.)

3.3.b. A right complete locally small category $\mathfrak{e}$ is generated by a full subcategory $a$ if and only if $\mathbb{C}$ is the full right closure of $a$.

The proof offers no difficulty. This notion, like all current one- 
sided notions of $a$ "sufficing" in $\mathcal{C}$, is not transitive; if $a$ generates $B$ and $B$ generates $\mathcal{C}, ~ Q$ need not generate $\mathcal{C}$ (5.7). It is the same (5.7) with a related notion going beyond wide-closure. A subcategory $B$ of $D$, or its set of objects, is called separating if for every coterminal pair of distinct maps $f, g: X \rightarrow Y$ in $D$ there exist $Z$ in a small part $B(Y)$ of $B$ and $h: Y \rightarrow Z$ in $D$ such that $h f \neq h g$. These relations are nevertheless of crucial importance, at least in the present state of knowledge, because of their role in the Special Adjoint Functor Theorem. For that theorem (3.12) we want an obvious lemma.

3.3.c. A locally small left small-complete category $D$ is separated by a subcategory $B$ if and only if every object of $D$ maps monomorphically into a small product of objects of B.

We will want the total closure properties: $B \subset \mathcal{C}$ is essentially left universal if its embedding is left conservative and every diagram in $B$ having a limit in $\mathcal{C}$ has a limit in $B$, left universal if it also contains all isomorphs and all isomorphisms of its objects. Because of the pullback criterion for monomorphisms

$$
(X \stackrel{f}{\rightarrow} Y \stackrel{f}{\leftarrow} X),
$$

a left universal subcategory of a left wide-complete category is left wide-closed.

We call a subcategory left conservative if its embedding functor is left conservative. The full left context of a subcategory $a$ of $\mathfrak{e}$ is the largest full subcategory with respect to which $a$ is left conservative, consisting therefore of all $X$ such that for every universal left conical extension $\left(L, \Phi: K_{L} \rightarrow D\right)$ of a diagram $D$ in $Q$ the induced map $\operatorname{Hom}\left(K_{X}, K_{L}^{\prime}\right) \rightarrow \operatorname{Hom}\left(K_{X}, D^{\prime}\right)$ (the primes mark the diagrams as considered in $C$ ) is invertible.

3.4. TheOREM. The full left context of any subcategory is right universal.

Proof. Let $R, \Phi: E \rightarrow K_{R}$ be a colimit of a diagram $E$ in the full left context $\beta$ of a subcategory $a(E: \mathcal{E} \rightarrow \mathcal{C}, E(\mathcal{E}) \subset \beta)$, and $L, \Psi$ : $K_{L}^{\prime} \rightarrow D$ a limit in $Q$ of a diagram $D: D \rightarrow Q ; K_{L}^{\prime}$ is, of course, the $L$ valued constant functor on D. Let $\Lambda: K_{R}^{\prime} \rightarrow I D$ be a left conical extension of $D$ considered in $\mathfrak{e}\left(I: a \subset \mathcal{e}, \Lambda \in \operatorname{Hom}\left(K_{R}, I D\right)\right)$. The maps $\Lambda_{X}(X$ in $D)$ and $\Phi_{Y}(Y$ in $\mathcal{E})$ have compositions $\Lambda_{X} \Phi_{Y}: E(Y) \rightarrow D(X)$, natural in $X$ and $Y$. (Formally, we are backing up the diagrams to a

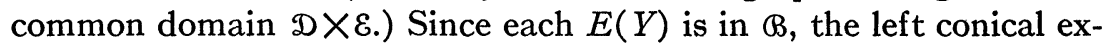
tension $(\Lambda \Phi)_{Y}: K_{E(Y)}^{\prime} \rightarrow I D$ defined by $\left((\Lambda \Phi)_{Y}\right)_{X}=\Lambda_{X} \Phi_{Y}$ factors uniquely across $L$, by $f_{Y}: E(Y) \rightarrow L$. Because of the uniqueness, 
$f: E \rightarrow K_{L}$ is right conical (natural). Hence there is a unique map $g: R \rightarrow L$ such that $g \Phi_{Y}=f_{Y}$ for all $Y$ in $\varepsilon$. Then $\Psi_{X} g \Phi_{Y}=\Lambda_{X} \Phi_{Y}$ for all $Y$, so that $\Psi_{X} g=\Lambda_{X}$. Retracing steps, no other $g$ can satisfy $\Psi_{X} g=\Lambda_{X}$ for all $X$ in $D ; \Psi$ is universal for $R$, and $R$ is in $B$.

3.5. Corollary. The intersection of the full left context of a subcategory $a$ with any full subcategory $\mathcal{F}$ containing $a$ is right universal in $\mathcal{F}$.

For the intersection is the full left context in $\mathcal{F}$. Note that the smallest full subcategory containing $a$ and satisfying 3.5 may be much larger than the right universal closure of $a$. Of course, the context need not contain $a$ if $a$ is not full.

3.6. Corollary. A left complete full subcategory is essentially left closed in its full right universal closure, and similarly for "small-complete," "strict-complete," "wide-complete."

For the embedding is left conservative. One could state more as in 3.5, but the whole Theorem 3.4 after all has a simpler statement. 3.6 will serve as a lemma for 3.7 .

A category $\mathfrak{C}$ is a completion of a full subcategory $a$ if $\mathfrak{C}$ is complete and every complete full subcategory of $\mathfrak{e}$ containing $Q$ is dense, i.e. equivalent in $\mathfrak{C}$ to $\mathcal{C}$. A normal completion of $a$ is a complete category $\mathfrak{e}$ containing $\mathfrak{a}$ as a full subcategory whose full left closure is $\mathfrak{C}$ and whose full right closure is $\mathfrak{C}$.

3.7. A normal completion is a completion. A completion $\mathfrak{e}$ of $a$ is normal if and only if every intermediate left or right complete full subcategory is dense; also, if and only if every object of $\mathfrak{e}$ is both an extremal quotient of a small coproduct of objects of a and an extremal subobject of a small product of objects of $a$. The embedding in a normal completion is left and right conservative.

Proof. If $\mathfrak{e}$ is a normal completion of $a$, then any left complete full subcategory containing $a$ has (also) right closure $\mathfrak{e}$; by 3.6 and 3.2 , it is equivalent to a left closed full subcategory containing $a$, so it is dense. The dual holds likewise and the converse is trivial, as are the implications " $Q$-multistrict" $\Rightarrow$ "multistrict" $\Rightarrow$ "extremal." The converse implications follow from 2.4 and from transporting defining relations $h_{\alpha} f=k_{\alpha} f$, for $h_{\alpha}: Y \rightarrow Z$, by monomorphisms $m: Z \rightarrow P$ where $P$ is a product of objects of $Q$. The last assertion follows from 3.4.

3.7.a. The same for strict-completion, small-completion, with appropriately restricted multistrict analyses.

3.7.b. If $\mathfrak{e}$ is a normal completion of $a$, the double regular envelope $a \rightarrow \operatorname{Co}(a, \mathfrak{u} !)$ can be extended to a full embedding of $\mathfrak{e}$, unique up 
to natural equivalence. The proof is a routine transfinite argument. The more interesting question of where the normal completions are in $\mathrm{Co}(a, \mathfrak{u} !)$ is for the sequel.

3.8. A normal completion of an ordinary category $a$ is equivalent to an ordinary category. In fact, if $a$ is a generating and separating full subcategory of $\mathfrak{C}$ then $\mathfrak{C}$ is locally small if $\mathfrak{Q}$ is and ordinary (up to equivalence) if $a$ is.

Proof. An extension $\mathbb{e}$ of a locally small category $Q$ is locally small whenever every object $X$ is both an epi quotient of a small coproduct of objects of $a$ and the dual; for every set $\mathfrak{e}(X, Y)$ then can be mapped one-to-one into a small product of sets $a\left(X_{\alpha}, Y_{\beta}\right)$. As for objects ( $Q$ ordinary now), there are only $\infty$ different small coproducts $C$ of objects of $Q$ and only $\infty$ different small products $P$ of objects of $a$. For each $C$ and $P$, consider the objects $X_{\alpha}$ for which there exist an extremal epimorphism $e_{\alpha}: C \rightarrow X_{\alpha}$ and a monomorphism $m_{\alpha}: X_{\alpha} \rightarrow P$. The compositions $f_{\alpha}=m_{\alpha} e_{\alpha}$ are only a small set of different maps in $\mathrm{e}(C, P)$, and $X_{\alpha}$ is determined up to isomorphism as the right image of $f_{\alpha}$. Thus there are only $\infty$ objects in all, i.e. $\mathcal{C}$ has an ordinary skeleton.

3.9. THEOREM. Every functor $F: \mathfrak{C} \rightarrow D$ has a pre-coadjoint $G: Q \rightarrow \mathfrak{C}$, which is unique up to natural equivalence and is left conservative in $D$.

Proof. By the definition of the domain $a$ for a pre-coadjoint, $L(F) L_{D} \mid A$ goes in to representable functors; thus it is naturally equivalent to a map into principal functors, which factors across $\mathbb{C}$. The result is unique up to a natural equivalence in $L_{\mathbb{C}}(\mathbb{C})$, which is (full and) isomorphic with $\mathcal{C}$. Given a diagram $E: \mathcal{E} \rightarrow \mathfrak{Q}$ such that $I E$ (where $I: a \subset D$ ) has a limit object $X$, assume (1) that $G E$ has a limit. Thus $h_{G E} K$ is representable. For $X$ to be in $Q$ requires representability of $h_{X} F$, or its natural equivalent $h_{I E} K^{\prime} F$. (Here $K: \mathfrak{e} \rightarrow \operatorname{Cat}(\mathcal{E}, \mathfrak{e})$, $K^{\prime}: D \rightarrow \operatorname{Cat}(\varepsilon, D)$.) We want a natural equivalence $\Phi: h_{I E} K^{\prime} F \rightarrow h_{G E} K$. We have a natural equivalence $\Psi: L(F)\left[L_{\mathscr{D}} \mid Q\right] E \rightarrow L_{\mathbb{C}} G E$. It remains to pick them to pieces and identify $\Phi$ with $\Psi . \Psi$ breaks down into maps $\Psi_{U}: h_{E(U)} F \rightarrow h_{G E(U)}(U$ in $\varepsilon)$, and these into $\Psi_{U T}: \operatorname{Hom}(F(T)$, $E(U)) \rightarrow \operatorname{Hom}(T, G E(U))$ ( $T$ in $\mathcal{C})$. For $\Phi$ we need functions $\Phi_{T}$ : $\operatorname{Hom}\left(K_{F(T)}^{\prime}, I E\right) \rightarrow \operatorname{Hom}\left(K_{T}, G E\right)$. Then for each $\alpha: K_{F(T)}^{\prime} \rightarrow I E$ we need $\Phi_{T}(\alpha): K_{T} \rightarrow G E$. We have $\alpha_{U}: F(T) \rightarrow E(U)$; put $\left[\Phi_{T}(\alpha)\right]_{U}$ $=\Psi_{U T}\left(\alpha_{U}\right)$. Then $\Phi_{T}(\alpha)$ is a map because $\Psi$ is natural in $U, \Phi_{T}$ is a function because so defined, $\Phi$ is a map because $\Psi$ is natural in $T$. $\Phi$ is invertible by formula, $\left[\Phi_{T}^{-1}(\beta)\right]_{U}=\Psi_{U T}^{-1}\left(\beta_{U}\right)$, where this is a twosided inverse by calculation.

For (2), we are given $X$ in $I(a)=a$, in this case, and required to 
show that the universal left conical extension $\lambda: K_{X}^{\prime} \rightarrow I E$, viz. $\theta_{X}\left(1_{X}\right)$ for a natural equivalence $\theta: h_{X} \rightarrow h_{I E} K^{\prime}$, induces a universal left conical extension $\mu$ of $G E$ by $\mu_{U}=G\left(\lambda_{U}\right): G(X) \rightarrow G E(U)$. Now $h_{X} F$

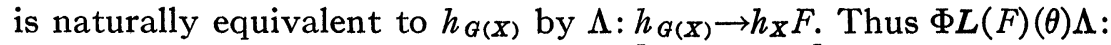
$h_{G(X)} \rightarrow h_{G E} K$ represents the limit, and $[\Phi L(F)(\theta) \Lambda]_{G(X)}\left(1_{G(X))}: K_{G(X)}\right.$ $\rightarrow G E$ is universal. Is it $\mu$ ? One must go back to the common source of $\Lambda$ and $\Psi$ in $\Omega: L(F) L_{D} \mid Q \rightarrow L_{\mathbb{C}} G ; \Lambda$ can be chosen as $\Omega_{\bar{X}}^{-1}$ and $\Psi$ so that $\Psi_{U}=\Omega_{E(U)}$ for all $U$. Then the verification is routine, and we omit it.

From 3.9, if both $\mathcal{C}$ and $D$ are left small-complete, then $a$ is left small-closed in $D$; thus $I: Q \subset D$ preserves monomorphisms and equalizers, whence $G$ does too, and $Q$ inherits one of the three stronger

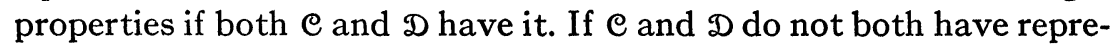
sentable limits, $G$ need not preserve equalizers; there is no difficulty in constructing examples (with $\mathfrak{C}$ or $\mathfrak{D}$ complete), as $\mathbb{Q}$ and $F(\mathbb{C})$ have little to do with each other. We note:

3.9.a. Every pre-coadjoint functor preserves monomorphisms.

It is trivial to check that this is (1) true and (2) not a consequence of 3.9.

3.10. Adjoint Functor Theorem. A left strict-closed functor $G: \mathbb{D} \rightarrow \mathfrak{C}$ has an adjoint if and only if every functor $h^{\mathbb{x}} G: \mathbb{D} \rightarrow \mathcal{U} !(X$ in e) is properly generated.

This is substantially Freyd's theorem [2]. Lawvere [11] made nontrivial improvements, and it is a trivial matter to adapt Lawvere's proof to the present definitions and conventions. Nevertheless the first draft of this paper contained a somewhat shorter proof. But the same proof is given in a preprint, Adjungierte Funktoren und primitive Klassen, which W. Felscher sent me in the meantime.

What will guarantee that the $h^{x} G$ are properly generated? One can restate the condition for some gain (3.11); one can put boundedness conditions on $D$ that are more stringent but more transparent (3.12). And then, one can do a little more if $G$ is a full embedding. The next two results are basically Freyd's, though our formulation of the Special Adjoint Functor Theorem 3.12 goes substantially further.

Relative to $G: \mathbb{D} \rightarrow \mathfrak{C}$, an object $X$ of $\mathfrak{C}$ is said to generate $Y \in \mathbb{D}$ if there is a mapping $f: X \rightarrow G(Y)$ such that whenever $f$ factors as $G(i) f^{\prime}$, where $i$ is a monomorphism into $Y, i$ is an isomorphism. Thus the objects generated by $X$ relative to the identity are its extremal quotients.

3.11. A left wide-closed functor $G: D \rightarrow \mathfrak{C}$ has an adjoint if $\mathfrak{C}$ is locally 
small and each object of $\mathfrak{e}$ generates only a small set of non-isomorphic objects of $D$ relative to $G$.

PRoOF (FREYD). If $\left\{Y_{\alpha}\right\}$ is a maximal set of nonisomorphic objects of $D$ generated by $X$, then $h^{x} G$ is generated by the small set of all maps from $X$ to all $G\left(Y_{\alpha}\right)$. For, given a map $g: X \rightarrow G(Z)$, let $i: Y \rightarrow Z$ be a monomorphic generator of the intersection of all subobjects of $Z$ generated by monomorphisms $j$ such that $G(j)$ is a left factor of $g$. Then $G(i)$ generates the corresponding intersection, so that $g$ factors as $G(i) f$. Hence $X$ generates $Y$.

There is a corresponding result where $D$ is merely left complete but each $X$ must have few maps to objects $G(Y)$ which fail to factor through proper strict subobjects, relative to $G$.

3.12. Special Adjoint Functor Theorem. A functor $G: D \rightarrow C$ with locally small domain and range has an adjoint if $G$ is left closed and $D$ is cogenerated by a small subcategory, or if $G$ is left wide-closed and $D$ is separated by a small subcategory.

Proof. We have expressed the hypotheses mostly in familiar terms (weaker, though, than the terms in [2]), but the assumption that $D$ is locally small has nothing to do with the theorem. Its purpose is to mediate the equivalences of 3.3.b, 3.3.c. In either case of the theorem, then, we have a distinguished small set of objects $Y_{\alpha}$ in $D$, and, since $\mathcal{e}$ is locally small, $\mathrm{U}_{\alpha} \mathfrak{e}\left(X, G\left(Y_{\alpha}\right)\right)=L=\left\{h_{\lambda}\right\}$ is small. Let $P$ be a product of the objects $Y_{\alpha}=E_{\lambda}$, each $Y_{\alpha}$ occurring once for each $h_{\lambda}: X \rightarrow G\left(Y_{\alpha}\right)$. Let $h: X \rightarrow G(P)$ be the mapping with $\lambda$-th coordinate $h_{\lambda}$ for all $\lambda$.

For the first part of the theorem, let $m: S \rightarrow P$ be an intersection of all the extremal monomorphisms $n$ into $P$ such that $G(n)$ is a left factor of $h$. Then $G(m)$ is itself a left factor of $h=G(m) j$. We claim $j$ generates $\mathfrak{e}(X, G())$. (Actually we need not appeal to 3.10 ; since $j$ factors through no proper strict subobject of $P$ relative to $G$, $G(f) j=G(g) j$ implies $f=g$. We need this later in the proof.) Consider any object $Y$ of $D$ and any element $y$ of $\mathfrak{e}(X, G(Y)) . Y$ is a multistrict subobject of a product $Q$ of factors $F_{\mu}$, each of which is a $Y_{\alpha}$; let $i=i_{\delta 0}: Y \rightarrow Q$ be a monomorphism with left multistrict analysis $\left\{Q_{\beta} ; i_{\gamma \beta}\right\}, Q_{\delta}=Y, Q_{0}=Q$. For each coordinate index $\mu$ of $Q$, the $\mu$-th coordinate of $G(i) y$ is some $h_{\lambda} \in L$; let $\lambda=\Lambda(\mu)$. Let $u: P \rightarrow Q$ be the mapping whose $\mu$-th coordinate is the $\Lambda(\mu)$-th coordinate projection of $P$, for each $\mu$. Then $G(u) h=G(i) y$, since they have the same coordinates. Thus we have $v_{0}=u m: S \rightarrow Q_{0}$ such that $G\left(v_{0}\right) j=G(i) y$. Moreover, we can factor $v_{0}$ across $i_{\delta 0}$ by the following induction. Having factored $v_{0}$ as $i_{B 0} v_{\beta}$, consider any pair of maps $a, b$ of $Q_{\beta}$ to $Z$ 
such that $a i_{\beta+1, \beta}=b i_{\beta+1, \beta}$. Since $G\left(i_{\beta 0}\right)$ is a monomorphism, we have $G\left(v_{\beta}\right) j=G\left(i_{\delta \beta}\right) y$. Now $G(a) G\left(i_{\delta \beta}\right) y=G(b) G\left(i_{\delta \beta}\right) y$, so $G\left(a v_{\beta}\right) j=G\left(b v_{\beta}\right) j$; therefore $a v_{\beta}=b v_{\beta}$, and $v_{\beta}$ factors as $i_{\beta+1, \beta} v_{\beta+1}, v_{0}=i_{\beta+1,0} v_{\beta+1}$. At a limit ordinal we merely note that $v_{0}$ belongs to the intersection of the preceding mono subobjects of $Q_{0}$. Finally, we have $v_{\delta}: S \rightarrow Y, y=G\left(v_{\delta}\right) j$. This completes the first part.

For the second part, let $m_{0}: S_{0} \rightarrow P$ be an intersection of all the monomorphisms $n$ into $P$ such that $G(n)$ is a left factor of $h$. Again $h=G\left(m_{0}\right) j$. Again we need to show that every $z: X \rightarrow G(Z)$ has $j$ as a right factor. This time $Z$ maps by a monomorphism $k$ into a product $Q$ of factors $F_{\mu}$, each of which is a $Y_{\alpha}$. Again the $\mu$-th coordinate of $G(k) z$ is some $h_{\lambda} \in L, \lambda=\Lambda(\mu)$. We have $u: P \rightarrow Q$ as before; now we note that $u$ factors as $l t$, where $t$ projects $P$ upon a partial product $P_{1}$ and $l: P_{1} \rightarrow Q$ is a monomorphism. As before, $G(l t) h=G(k) z=r$. Thus $k$ and $l$ are two monomorphisms into $Q$ such that $G(k)$ and $G(l)$ are left factors of $r$; there is an intersection $i: I \rightarrow Q$, with monomorphisms $a: I \rightarrow Z, b: I \rightarrow P_{1}$, and a mapping $c: X \rightarrow G(I)$, such that $k a=l b=i, G(i) c=r$. In particular, since $G(l)$ is monomorphic, $G(b) c$ $=G(t) h$. Now note that $P$ is a product of $P_{1}$ and a remaining factor $P_{2}$, with second coordinate projection $s: P \rightarrow P_{2}$. Let $d$ be the mapping from $X$ to $G\left(I \times P_{2}\right)=G(I) \times G\left(P_{2}\right)$ with first coordinate $c$ and second coordinate $G(s) h$; let $n$ be the mapping $b \times 1$ from $I \times P_{2}$ to $P_{1} \times P_{2}$ $=P$. Then $n$ is a monomorphism, since its factors are. $G(n) d: X$ $\rightarrow G\left(P_{1} \times P_{2}\right)$ has first coordinate $G(b) c=G(t) h$ and second coordinate $G(s) h$; so $G(n) d=h$. Therefore $n$ is a left factor of $m_{0}=n e$. If $f: I \times P_{2}$ $\rightarrow I$ is the first coordinate projection, we now have $G(k) z=G(l t) h$ $=G\left(l t m_{0}\right) j=G(l t n e) j=G(l b f e) j=G(k a f e) j$. Since $G(k)$ is monomorphic, $z=G(a f e) j$, as required.

As Kan observed in 1958 [11], the limit functors that one wants to represent for completeness are closely related to the functors one wants to represent for adjoints, and small-completeness can be defined in terms of adjoints. By 1964 Freyd [2] and I [8] had noticed that there is a crossover, left complete $\rightarrow$ left adjoint $\rightarrow$ right complete (saving boundedness problems. Note that [8] contains no adjoint functor theorem, only fragments relevant to this question. Both [2] and [8] contain substantial kernels of the theorem below, 3.15). An amalgamation of a family of objects $X_{\alpha}$ is an object $Y$ with maps $f_{\alpha}: X_{\alpha} \rightarrow Y$ such that no strict or representable proper contravariant ideal in $Y$ includes all $f_{\alpha}$ in its values. From [8], an ordinary left wide-complete category in which each small family of objects has only a small set of amalgamations (up to isomorphism) is right strictcomplete. Having additional concepts available, we can note that 
"locally small" can replace "ordinary," with the same proof, and that the category must be right complete because extremal quotients are simply amalgamations of a one-member family.

3.13. Lemma. In any category, a family of maps $f_{\alpha}: X \rightarrow Z_{\alpha}$ can be factored in at most one way (up to isomorphism) as $g_{\alpha} q$, where $q: X \rightarrow Y$ is a multistrict epimorphism and every two different maps into $Y$ have different compositions with some $g_{\alpha}$. In a category with finite limits, a family of maps $f_{\alpha}: X_{\alpha} \rightarrow Z$ can be factored in at most one way as $i h_{\alpha}$, where $\left\{h_{\alpha}\right\}$ is an amalgamation and $i$ a monomorphism.

REMARK. The obvious name for $\left\{g_{\alpha}\right\}$ is "separation."

The first part of 3.13 is proved by routine transfinite induction. For the second part, $i$ generates a minimal subobject including all $f_{\alpha}$, and given finite limits, it is the smallest.

3.14. Lemma. A locally small category $\mathfrak{C}$ is well-powered if it has finite limits and a small generating subcategory. If e has a small separating subcategory, each object has only a small set of multistrict quotients; if it is also left small-complete, then each small family of objects has only a small set of amalgamations.

REMARK. The two parts of 3.13 have a union which can be used for a small refinement of 3.14. We have no other use for the concept required to state it, so omit it.

Proof. Take the last assertion first. An amalgamation $\left\{f_{\alpha}: X_{\alpha} \rightarrow Y\right\}$ induces a one-to-one function from any set $\operatorname{Hom}(Y, A)$ into the Cartesian product of $\operatorname{Hom}\left(X_{\alpha}, A\right)$. If $\left\{X_{\alpha}\right\}$ is small and a small set of $A$ 's separate, we have one small product $Z$ into which every such $Y$ maps monomorphically, $i: Y \rightarrow Z$. By 3.13, $\left\{i f_{\alpha}\right\}$ determines $Y$, and there are only a small number of possibilities. The second assertion is proved in the same way, using the coordinates of $i$ and the other half of 3.13. For the first assertion, given an object $Z$, every mono subobject of $Z$ is an amalgamation of generators $X_{\alpha}$, and a fixed small family can be used since $Y \subset Z$ induces $\operatorname{Hom}(X, Y) \subset \operatorname{Hom}(X, Z)$. Again 3.13 finishes it.

3.15. Theorem. A locally small left wide-complete category with a small separating subcategory is right complete.

From 3.14 and the result quoted from [8]. Also from 3.14, the assumption that $\mathcal{C}$ is left small-complete with small generating and separating sets is stronger. It implies more, 4.8 below. We can also deduce right completeness, and more, if $\mathfrak{e}$ is left small-complete and has a small right adequate subcategory (4.7). There is an obvious 
"average" hypothesis between 3.15 and 4.7, namely left completeness and a small cogenerating subcategory. This, too, implies right completeness. The category is right small-complete by Kan's argument [10] and 3.12. (Kan's argument: the colimit functors $h^{D} K$ to be represented are the constituents of a left adjoint for $K: \mathfrak{e}$ $\rightarrow$ Cat $(D, C)$. One readily checks that $K$ is left and right conservative.) It is then co-well-powered by 3.14 .

We are especially interested in adjoints of embedding functors. A subcategory $\mathfrak{D}$ of $\mathfrak{C}$ is called reflective (coreflective) if its embedding functor has a left (right) adjoint. 3.10-3.12 show that left closed subcategories satisfying boundedness conditions are reflective. There are many reflective subcategories (e.g. in abelian groups; see 5.5) that are nowhere near left closed. However:

3.16. A reflective full subcategory of any category is essentially left universal and is a retract.

Proof. Let $I: Q \rightarrow \mathfrak{C}$ embed a full subcategory and let $R: \mathfrak{e} \rightarrow \mathfrak{Q}$ (the reflector) be left adjoint to $I . R$ takes each $X$ of $\mathfrak{e}$ to $R(X)$ in $a$ such that for each $Y$ in $Q$ there is a one-to-one correspondence $\Phi_{X Y}$ of $\mathfrak{e}(X, Y)$ with $\mathfrak{Q}(R(X), Y)=\mathfrak{e}(R(X), Y)$. In particular, a certain map $r_{X}: X \rightarrow R(X)$ corresponds to $l_{R(X)}$, and since $\Phi$ is natural, there is for each $f: X \rightarrow Y(Y$ in $a)$ a unique $f^{\#}=\Phi_{X Y}(f)$ such that $f^{\#} r_{X}=f$. If $X$ is a limit of any diagram $I D: D \rightarrow Q \rightarrow \mathcal{C}$, via a natural transformation $\Psi: K_{X} \rightarrow I D$, then one readily checks that replacing each $\Psi_{Z}: X \rightarrow D(Z)$ with $\Psi_{Z}^{\#}: R(X) \rightarrow D(Z)$ presents $R(X)$ as a limit of $I D$. Thus $a$ is essentially left universal. Finally, for $X$ in $a, r_{X}$ and $1^{\#} X$ are inverse isomorphisms, so that there is a functor $1^{H}: Q \rightarrow Q$ such that $1^{\sharp} R: \mathbb{C} \rightarrow \mathbb{Q}$ is a retraction.

Let us take a brief further look at general adjoint functors. There is a sort of reduction to embedding functors and their adjoints, announced by Freyd [2] but considerably overstated by him. First the conclusion: under hypotheses like those of 3.12 but applied to both categories, a pair of adjoint functors $\mathfrak{C} \rightleftarrows \mathbb{D}$ can be factored across at least four "images" so that the factors are embeddings and (left or right) adjoints of embeddings. (One might say that Freyd understated.) Unfortunately, adjoints of embeddings are only distant relatives of epimorphisms. For each of the factorizations to be described, one of the embeddings is full, so that its adjoint may be chosen to be a retraction onto.

Freyd used a preliminary transformation which he called an assumption: the right adjoint $G: \mathbb{D} \rightarrow \mathfrak{C}$ is one-to-one on objects. Evidently we can secure this, without affecting adjoints, by embedding $\mathfrak{C}$ densely in a category having sufficiently many copies of each ob- 
ject and replacing $G$ with a suitable natural equivalent. In this situation the set of all $G(X)$ and all $G(f)$ forms a subcategory of $\mathfrak{e}$, the quotient $D / G$; and $G$ is factored into $Q: D \rightarrow D / G$ and $I: D / G \rightarrow \mathcal{C}$. Arguments sketched on page 83 of [2] show that $Q F$ and $I$ are again adjoint functors; $D / G$ is reflective in $\mathcal{C}$.

Freyd went on to state that $F I$ and $Q$ are adjoint. This is false as of ten as not. Since adjoints compose, it would require $G=I Q$ to have the adjoint $F I Q F=F G F$, which must then be naturally equivalent to the given adjoint $F$. If $G$ is a standard grounding, $F$ a free-object functor, this means that every free object is freely generated by all of its elements. In fact the quotient is not one of the places at which adjoint factorization is possible; if $G$ is $h^{T}$ where $T$ is the group $Z_{2}$, the factor $Q$ does not preserve the equalizer of the two maps from $Z_{4}$ to $T$. There is a similar result-one good factor, without any restriction on the categories-at $\hat{G}(D)$, the smallest full subcategory of $\mathfrak{e}$ containing $G(D)$. Moreover, applying these remarks to $F$, we get two other places at which half of the factorization can be done.

If factorizations are arranged in order as in (2.7), $D / G$ gives the longest embedding one could hope to use. One of the factors below (3.18) is the longest possible adjoint of an embedding; thus when $D / G$ does not work, it is too far off center. The full subcategory $\hat{G}(D)$ is much more central, and the commonest case in which it works is when it is dead center (coinciding with $\hat{F}(\mathfrak{C})$ ). This notion of center might repay study; what we have is a partially ordered set with a partially defined involution.

How far out can one go and still get embeddings?

3.17. ThEOREM. If $F: \mathfrak{C} \rightarrow \mathfrak{D}, G: D \rightarrow \mathfrak{C}$ are adjoint functors, $F$ is an embedding if and only if $\hat{G}(D)$ is separating.

Proof. If $\hat{G}(D)$ is separating, two different maps $f_{i}: C \rightarrow C^{\prime}$ yield different maps $h f_{i}: C \rightarrow G(D)$, which correspond adjointly to different maps $k_{i}: F(C) \rightarrow D$. Also $1: G(D) \rightarrow G(D)$ corresponds to $j: F G(D) \rightarrow D$, and $1 h f_{i}$ to $j F\left(h f_{i}\right)$, by naturality. Thus $[j F(h)] F\left(f_{1}\right) \neq[j F(h)] F\left(f_{2}\right)$, $F\left(f_{1}\right) \neq F\left(f_{2}\right)$. Conversely, suppose $F$ is an embedding ( $G$ a coreflector). By a simple (known [2]) computation, every $F(C)$ is a retract of $F G F(C)$, by $e: F G F(C) \rightarrow F(C)$ corresponding adjointly to $1: G F(C)$ $\rightarrow G F(C)$, and $F(m): F(C) \rightarrow F G F(C)$, where $m$ corresponds adjointly to $1: F(C) \rightarrow F(C)$. Then $m=F(m)$ is monomorphic in $D$, so a fortiori in $\mathfrak{e}$.

3.18. Theorem. Suppose $G: D \rightarrow C$ is a coadjoint functor with locally small domain and range, and either $D$ has a small cogenerating subcate- 
gory and $\mathfrak{D}$ and $\mathfrak{C}$ are left complete, or $\mathfrak{D}$ has a small separating subcategory and $\mathfrak{D}$ and $\mathfrak{C}$ are left wide-complete. Then $G$ can be factored into a coadjoint of an embedding and a coadjoint embedding.

Proof. On the second alternative hypothesis, note that since $G$ preserves products and monomorphisms, it takes a small separating subcategory of $\mathscr{D}$ to a small separating subcategory $Q$ of $\hat{G}(D)$. The full subcategory $\mathfrak{g}$ of $\mathfrak{C}$ on all wide subobjects of products of objects of $Q$ is left wide-closed, so $\mathscr{I} \subset \mathcal{C}$ is a coadjoint embedding by 3.12 . The adjoint of $\mathfrak{D} \rightarrow \mathscr{I}$ is the restriction to $\mathscr{I}$ of the adjoint of $G$, an embedding by 3.17 . The other alternative works similarly.

The boundedness conditions in 3.18 are annoying, since (unlike 3.12) it seems likely that they are nearly irrelevant. One can at least provide other alternatives, since the dual approach, factoring $F$ at these places instead of $G$, uses different constructions. We omit that.

3.19. Theorem. Let $\mathfrak{e}$ be a locally small, small-complete category that is left or right extremal-complete, and a a small full subcategory. Then a has a normal completion in $\mathfrak{e}$, upon which $\mathfrak{e}$ can be retracted by composing a reflector and a coreflector.

3.20. THEOREM. Every locally small completion of a small category is a normal completion and is well-powered and co-well-powered.

These results leave open the possibility that a locally small minimal small-complete extension can be manufactured which lacks these properties. I have no example; but let us spell out a proposition on that.

3.19.a. Suppose a is a small full subcategory of a locally small, smallcomplete category $\mathfrak{C}$. Then $\mathfrak{C}$ is a normal completion of $\mathfrak{a}$, or $\mathbb{a}$ is contained in a small-complete reflective or coreflective full subcategory that is not dense, or else some object of $\mathfrak{C}$ has a large (or extraordinary) set of multistrict subobjects and some object of $\mathfrak{e}$ has a large set of multistrict quotients. In the latter case $\mathfrak{a}$ does not separate or coseparate $\mathfrak{C}$.

Proof OF 3.19. In case $\mathcal{C}$ is left extremal-complete, let $B$ be the full left closure of $a$. By the Special Adjoint Functor Theorem, $B$ is reflective. By 3.14, $B$ is co-well-powered; so $B$ is right complete. Then dually, the full right closure $\varepsilon$ of $Q$ in $B$ is complete and co-reflective in $\beta$, and $\mathcal{E}$ is a normal completion of $a$. The other case is dual.

Now 3.20 follows from 3.19 and 3.14. So does 3.19.a.

Define the reflectivizer of a subcategory $\mathbb{B}$ of $\mathfrak{C}$ as the domain of the pre-adjoint of the embedding $I: B \subset \mathfrak{C}$; that is, the full subcategory on the objects $X$ for which $h^{\mathrm{x}} I$ is representable. Evidently the reflectivizer is contained in the full left context of $B$.

3.21. The reflectivizer of a right complete subcategory a of a right 
complete category is right closed, and its intersection with any right complete full subcategory $B$ containing $Q$ is right closed in $B$. If $\propto$ is full, it is essentially left universal in its full right closure. If all six occurrences of "complete," "closed," "closure" are modified by "small-," "strict-," or "wide-," the result still holds.

ProOF. Most of this restates remarks after 3.9. For the full subcategory $B$ containing $Q$, its intersection with the reflectivizer is the reflectivizer of $a \subset B$. If $Q$ is full, the reflectivizer contains it and its full right closure, and by $3.16 a$ is essentially left universal there.

Summarizing 3.6 and 3.21 :

3.22. A left complete full subcategory is always essentially left closed in its right universal closure; if the containing category is left complete, it is essentially right universal in its left closure.

3.23. In a left complete category such that every left closed full subcategory is a retract, every left complete full subcategory is a retract.

For its coreflectivizer is a retract.

But what categories are these? We shall not come near to any satisfactory answer, yet there are detachable parts of the problem. First, let us ask that the left closed full subcategories be reflective; there exist left closed, full, non-reflective retracts (5.6), but maybe they do not occur in familiar examples. (Maybe their existence even implies that some other left closed full subcategory is not a retract.) Next we can try to take a limit of reflections provided by 3.12 .

3.24. In a locally small left complete category, given a mapping $r: X \rightarrow R$, there exists a reflective full subcategory $Q$ including $R$ such that $r$ represents $Q(X$,$) if and only if for every g: X \rightarrow R$ there is a unique $f: R \rightarrow R$ such that $f r=g$.

We omit the routine check; $Q$ is the full left closure of $R$ and 3.12 applies. Call these mappings $r$ reflection mappings.

3.25. If $f g$ is a reflection mapping and $g$ is an epimorphism then $f$ is a reflection mapping; if also $f$ is an extremal monomorphism, then $g$ is a reflection mapping. The full left closure of the union of a small set of reflective full subcategories is reflective.

We omit the three routine checks. For the second one, one considers right images.

It follows that if each object is the domain of only a small set of different reflection mappings $(r: X \rightarrow R$ and $s: X \rightarrow S$ being considered different if they are not related by an isomorphism $R \rightarrow S$ ), then every left closed full subcategory is reflective. This seems a reasonable condition, but little is known about it. By 3.25, one can factor out epimorphisms. Define a (reflective) frame of an object $X$ as an equivalence class of maps $r: X \rightarrow R$ which are at once reflections and ex- 
tremal monomorphisms, equivalents being left multiples by isomorphisms.

3.26. In a locally small left complete category, if every object has only a small set of wide quotients and a small set of frames, then every left closed full subcategory is reflective. Consequently every left complete full subcategory is a retract (coreflection of a reflection) and is right strictcomplete if the given category is right strict-complete.

I do not know whether $Z$ has a small set of frames in the (small abelian) groups. It has more than continuum-many, by taking weak or strong direct sums of suitable families of subgroups of $Q$.

Suppose we assume (third) that the left closed full subcategories $B$ of $\mathfrak{e}$ are right small-complete. This amounts to assuming that twothirds of the problem is solved, for a $B$-coproduct of objects $B_{\alpha}$ is the same thing as a reflection of their $\mathfrak{C}$-coproduct, and similarly for coequalizers. At any rate the assumption this time is a necessity rather than an over-strong convenience, and the remaining piece of the problem is still difficult, yet accessible. From 3.21, a left closed right complete subcategory $B$ is reflective if $\mathcal{C}$ is right complete and every object of a generating set has a reflection in $B$. If $\mathfrak{C}$ is a primitive category of algebras, the free algebra on one generator generates it, by right small-closure; and we have reduced (for full, right small-complete $B$ ) to determining the frames of cyclic algebras. In lattices, for instance, cyclic $\Rightarrow$ injective (which always implies triviality of frames), and there is only the question whether every left closed full subcategory is right small-complete. I have no idea. In distributive lattices it is trivial; this is an example of a "simple" category, where every object except some trivial ones (having fewer than 2 elements) is a generator and a cogenerator. In the compact (Hausdorff) spaces, every non-empty space generates, and of course there is a cogenerator (moreover, a right adequate object [6]). The one-sided simplicity makes every left complete full subcategory left closed, except the full subcategory on the empty space. Thus every complete full subcategory of the compact spaces is a retract. That sounds like a theorem; but if one pulls out the proof and looks at it, one sees that "right smallcomplete" is enough and the proof is trivial. It (like the literature) tells us nothing about reflective frames of a two-point space.

4. Regular completion. Let us call a category left transcomplete if every diagram in it has a limit. Note that this does not imply even left strict-completeness, for a diagram cannot be a proper class but an object might have a proper class of strict subobjects. Hence we want some boundedness lemmas for functor categories.

Call a functor $F$ in $\operatorname{Cat}\left(\mathfrak{e}^{*}, \mathfrak{u} !\right)$ separated if it is a term of a sepa- 
rated couple, or equivalently, it maps monomorphically into a product of principal functors. The equivalence depends on the easily checked fact that a monomorphism $\Phi$ of functors must be one-to-one, i.e. every function $\Phi_{X}$ is one-to-one. That fact makes the next lemma obvious. In these lemmas, and wherever Cat is applied, $\mathfrak{e}$ has only a set of objects.

4.1. Every full subcategory of $\mathrm{Cat}\left(\mathfrak{C}^{*}, \mathfrak{U} !\right)$ containing the principal functors is set-powered.

4.2. Every full subcategory of separated functors containing the principal functors is co-set-powered.

Proof. If $F$ maps epimorphically to $G$, every $\operatorname{Hom}\left(G, h_{X}\right)$ has cardinal number at most that of $\operatorname{Hom}\left(F, h_{X}\right)$. Thus the separated wide quotients of $F$ are among the subfunctors of a certain product of principal functors, and there are only a set of them.

Let us use the symbol $D$ for the isomorphic copy of $\mathcal{C}$ consisting of the principal functors in $\operatorname{Cat}\left(\mathfrak{e}^{*}, \mathcal{U} !\right)$ and their maps. The left regular transcompletion $\mathscr{L} !$ of $\mathfrak{C}$ is the left transclosure of $\mathscr{D}$, i.e. the full subcategory on the D-multistrict subobjects of products of objects of $D$. We call the objects of $\& !$ stable cogroundings. What they are stable under is the reflector $Q: \operatorname{Cat}\left(\mathfrak{e}^{*}, \mathfrak{u}\right) \rightarrow \mathscr{L} !$. The existence of this reflection follows from the Special Adjoint Functor Theorem, by a reinterpretation replacing "small set" by "set" throughout; the required left completeness of the domain $\&$ ! follows from 4.1 .

4.3. THEOREM. The left regular transcompletion of a category $\mathfrak{e}$ with a set of objects is reflective in $\mathrm{Cat}\left(\mathfrak{e}^{*}, \mathfrak{u}\right.$ !) and left and right transcomplete and wide-complete. The embedding of $\mathfrak{C}$ in it is left and right conservative.

Proof. We just showed that $\&$ ! is reflective. By 3.16 , it is a retract; by 3.1 , it is transcomplete; by 4.1 and 4.2 , it is wide-complete. The last assertion follows from 3.4.

It will be convenient to have an explicit description of the reflector $Q$ and a test for stability. ${ }^{7}$ Consider the evaluation $\epsilon: F \rightarrow F^{* *}$; it is defined in the obvious way [6], which is equivalent to deriving it from the natural coupling of $F$ and $F^{*}$ by the dual of 1.1. Take the $D$-dominion $D$ of $\epsilon$, i.e. the smallest containing $D$-sesquistrict subobject, and the factor maps $q: F \rightarrow D, i: D \subset F^{* *}$. Every map $s$ from $F$ to a principal functor $h_{Y}$ is an element of $F^{*}(Y)$ and is divisible by $\epsilon$ by evaluation; define $\bar{s}_{X}(t)=t_{Y}(s)$ for each $t \in F^{* *}(X)$, and check $\bar{s} \epsilon=s$. Thus $\bar{s} i q=s$. Since $D$ is the dominion, $\bar{s} i$ is the only solution $u$ of $u q=s$. Then every map from $F$ to a product $P_{0}$ of principal functors is uniquely divisible by $q$. Given a $D$-multistrict analysis

\footnotetext{
${ }^{7}$ The construction is a specialization of the dual of half of 3.18 .
} 
$\left\{P_{\alpha} ; f_{\alpha \beta}\right\}$ and a map $s: F \rightarrow P_{\alpha}$, having $u_{\beta}: D \rightarrow P_{\beta}$ such that $u_{\beta} q=f_{\alpha \beta} s$, the defining relations for $f_{\beta+1, \beta}$ come from maps into principal functors, and since $u_{\beta} q$ satisfies them, so does $u_{\beta}$. Hence $u_{\beta+1}$; similarly at limit ordinals, and $D$ is the stable reflection of $q$. (The lemma here is, of course, that a proposed reflection need only be checked for maps into a set of (improper) cogenerators.)

So stable functors are limits of limit functors. They need not be limit functors (5.8).

There is another conclusion from this argument that we shall want in the midst of the next theorem; given a map $r: F \rightarrow G$ in $\operatorname{Cat}\left(\mathfrak{e}^{*}, \mathfrak{u}\right.$ !), if $F$ is stable and $r^{*}: G^{*} \rightarrow F^{*}$ is invertible, then $r$ has a left inverse. For the inverse of $r^{*}$ takes (coordinate) maps $s$ from $F$ to principal functors to solutions $u$ of $u r=s$, and the argument above (reduced now to two steps) applies again.

The reflector $Q$ yields a good factorization of $K_{1}$. This is deducible from 3.18, but let us treat it directly. (1st) Given $\Phi: F \rightarrow G, \Psi: F \rightarrow G$, if $Q(\Phi)=Q(\Psi)$ then $K_{1}(\Phi)=K_{1}(\Psi)$; for $K_{1}(\Phi)$ and $K_{1} Q(\Phi)$ are the sides of a commutative rectangle whose ends are isomorphisms $K_{1}(q)$. (2nd) If $Q(\Phi) \neq Q(\Psi)$, then some map from $Q(G)$ to a principal functor separates them, and $K_{1} Q(\Phi) \neq K_{1} Q(\Psi)$. Therefore $K_{1}$ factors across $Q$ and the other factor $J_{1}: \mathfrak{L} ! \rightarrow \operatorname{Cat}(\mathcal{C}, \mathcal{U} !)$ is a dual embedding. Moreover, it is trivial to check that $Q K_{2}$ and $K_{1} \mid \& !$ are adjoint on the right.

Let us define the left regular left completion $\mathfrak{L}$ of $\mathfrak{e}$ as the left closure of $\mathscr{D}$ in $\mathscr{L}$ ! (or equivalently in Cat $\left(\mathfrak{C}^{*}, \mathfrak{u} !\right)$ ); the left regular completion $\mathfrak{L}_{\infty}$ is defined inductively in $\mathscr{L}$ ! by putting $\mathscr{L}=\mathfrak{L}_{1}, \mathfrak{L}_{2 \alpha+1}$ the left closure of $\mathfrak{L}_{2 \alpha}, \mathfrak{L}_{2 \alpha+2}$ the right closure of $\mathfrak{L}_{2 \alpha+1}$, and $\mathfrak{L}_{\lambda}$ (for limit numbers $\lambda$ ) the union of the preceding $\mathscr{L}_{\alpha}$. This hog-wild method of completing is not successful in general, but we are not in general.

4.4. ThEOREM. The left regular completion $\mathfrak{L}_{\infty}$ of $\mathfrak{C}$ is a completion of e. Every left complete full subcategory of Cat( $\mathfrak{C}^{*}, \mathfrak{u}$ !) containing $D$ is essentially left closed. Every right complete full subcategory of $\$$ ! containing $D$ is essentially right closed. Thus $\mathfrak{L}_{\infty}$ is the unique completion of $\mathfrak{C}$, up to equivalence, in $\mathbb{\&}$ !.

If $\mathfrak{C}$ is small, its left regular left completion $\mathfrak{L}$ is already right complete. $\mathcal{L}$ is well-powered and co-well-powered and is a (normal) completion of $\mathfrak{e}$, the sole completion (up to equivalence) in $\mathrm{Cat}\left(\mathfrak{C}^{*}, \mathfrak{u}\right.$ !).

Proof. Completeness of $\mathscr{L}_{\infty}$ follows from the fact (and its dual) that $\&_{2 \alpha}$-multistrict subobjects of objects in $\mathfrak{L}_{2 \alpha+1}$ are the same as their $\mathbb{D}$-multistrict or $\&$ !-multistrict subobjects, since everything maps monomorphically into a product of (no matter how many) objects of $D$. The second assertion of the theorem is clear from the consideration that every object $X$ in the category of cogroundings is 
determined by $\mathscr{D}(, X)$; thus if $X$ is a limit of a given diagram it is the limit. Next consider a colimit $F$ of a diagram $D$ in a right complete full subcategory $\mathfrak{T}$ of $\mathscr{L}$ ! containing $D$. Compare with the $\mathscr{L} !-$ colimit $F_{0}$ of $D$. The right conical extension of $D$ by $F$ is connected to the universal right conical extension by a map $r: F_{0} \rightarrow F$ which induces a natural equivalence of $\operatorname{Mr}(F$,$) and \operatorname{mC}\left(F_{0}, \quad\right)$. Thus $r^{*}$ is invertible, and since $F_{0}$ is stable, $F_{0}$ is a retract of $F ; F_{0}$ is in $T$. The first paragraph is proved.

The rest of the theorem follows from 4.3, 4.1, 4.2, with a reinterpretation replacing "set" by "small set"; and 2.1.a.

We call a category $\mathfrak{e}$ injective if it is locally small and is a retract of every locally small category $\mathcal{E}$ in which $\mathfrak{C}$ is fully embedded so that $\varepsilon$ contains just one object not in $\mathcal{C}$. Let us show, if we can, that this implies as much as one could reasonably ask.

For non-full embeddings, $u$ can be embedded in itself by $X \rightarrow X \times X$, and there is no retraction because symmetric groups are not direct sums. The same argument applies to, for instance, groups and vector spaces. (I do not know if the suggested retraction property is equivalent to being wide-complete and trivial, where triviality means no two coterminal maps exist.)

4.5. THEOREM. Every functor from a small full subcategory of an ordinary category to an injective category $\mathfrak{e}$ can be extended; and $\mathfrak{e}$ is a retract of every locally small category $\mathcal{E}$ in which $\mathfrak{e}$ is fully embedded so that $\mathcal{E}$ contains at most $\infty$ objects not in $\mathbb{C}$.

Both proofs are routine exercises, if one recalls the induction scheme for the Hahn-Banach theorem.

We will not in fact construct retractions that way; so we may as well mention a stronger, illegitimate, but not rare, property. $\mathfrak{e}$ is extraordinary-injective if it is locally small and is a retract of every locally small category in which $\mathfrak{e}$ is fully embedded.

4.6. EXA'MPLE. Not every ordinary grounding of a full subcategory of a trivial ordinary category can be extended.

This is with the other examples, in $\$ 5$. Every properly generated grounding of a full subcategory of an ordinary category can be extended [6]. Thus there seem to be interesting boundedness questions in the extensions of functors with injective range, which are not our present concern.

4.7. THEOREM. Every locally small right small-complete category having a small left adequate subcategory is ordinary, well-powered, left complete, right strict-complete and extraordinary-injective.

Proof. Such a category $\mathfrak{e}$, having a small left adequate subcate- 
gory $a$, is fully embedded in $\operatorname{Cat}\left(a^{*}, \mathfrak{u}\right)$ by subregular representation, and therefore it is ordinary. As it contains the principal functors, 4.1 shifted to small sets implies that $\mathfrak{e}$ is well-powered. By 3.21 and 2.1.a, $\mathcal{e}$ is reflective in $\operatorname{Cat}\left(a^{*}, \mathfrak{u}\right)$; thus it is right strict- and left wide-complete. If $\mathfrak{C}$ is fully embedded in a locally small category $\mathcal{E}$, subregular representation and reflection $\varepsilon \rightarrow \operatorname{Cat}\left(a^{*}, \mathfrak{u}\right) \rightarrow \mathcal{C}$ yield a retraction. [8].

4.7 applies to primitive and quasi-primitive categories of algebras

4.8. Theorem. A left complete category having small generating and separating sets and only a set of objects-in particular, a normal completion of a small category-is extraordinary-injective.

Proof. Such a category $\mathcal{C}$ is locally small by 3.8 (set of objects used here), complete by 3.14 and 3.15. Given a full embedding of $\mathfrak{e}$ in a locally small category $\mathcal{E}$, we set about retracting by subregular representation $\mathcal{E} \rightarrow \operatorname{Cat}\left(\mathfrak{e}^{*}, \mathfrak{u}\right) \subset \operatorname{Cat}\left(\mathfrak{C}^{*}, \mathfrak{u} !\right)$, reflect into $\mathcal{L}$ !, and must show that the resulting cogroundings are representable. Consider any $F$ in $\operatorname{Cat}\left(\mathfrak{e}^{*}, \mathcal{u}\right)$. In a value of the conjugate $F^{*}(Z)$ $=\operatorname{Hom}\left(F, h_{Z}\right)$, suppose $\alpha \neq \beta$. Then $\alpha_{Y} \neq \beta_{Y}$ for some $Y$, meaning $\alpha_{Y}(y)$ and $\beta_{Y}(y)$ are distinct maps $Y \rightarrow Z$ for some $y \in F(Y)$. In a small coseparating (not to mention generating) set there must be an object $X$ such that for some $f: X \rightarrow Y, \alpha_{Y}(y) f \neq \beta_{Y}(y) f$. That is, $\alpha_{X}$ and $\beta_{X}$ have different values at $F(f)(y) \in F(X)$. The union of all these $F(X)$ is small, as also for the $h_{Z}(X)$; so $F^{*}$ is small-valued. We also assumed a small separating set, so we may conclude that $F^{* *}$ and $Q(F)$ are small-valued. Now $Q(F)$ is a small-valued multistrict subfunctor of some product of principal functors $h_{z}, Q(F) \subset P$; we need only show that a small number of $Z$ 's suffice. (As repeated coordinate functions can be ignored, this puts $Q(F)$ in $\mathscr{L}$, which consists of representable functors by 4.4.) Note that the embedding of $\mathcal{C}$ in $\mathscr{L} !$ is left and right conservative by 4.3 . Take a small generating set $S$ of $X$ 's and form the coproduct $\Sigma$ in $\mathfrak{C}$ of copies of $X$ 's each indexed by $Q(F)(X)$. There is a universal map $\gamma: h_{\Sigma} \rightarrow Q(F)$, whose $p$ th coordinate $\pi$ for each index $p \in Q(F)(X)$ is determined by $\pi\left(1_{X}\right)=p$. Moreover, $\gamma$ is an extremal epimorphism in $\mathcal{L}$ !, i.e. $S$ generates, albeit improperly. For if $\gamma$ has left image $G, \Sigma \rightarrow G \subset Q(F)$, we shall find that every map $\alpha: h_{T} \rightarrow Q(F)$ factors through $G$, i.e. $\alpha\left(1_{T}\right) \in G(T)$; and every element of every $Q(F)(T)$ has the form $\alpha\left(1_{T}\right)$. To prove this, represent $T$ as an extremal quotient of a coproduct $U$ of objects of $S$. We get an extremal epimorphism $q: h_{U} \rightarrow h_{T}$, and $\alpha q$ factors through $G$ since $\gamma$ is universal (all its restrictions to "summands" $h_{X}$ of $h_{U}$ factor through $G)$. Now $i j: h_{U} \rightarrow G \rightarrow Q(F)$ is $\alpha q=f$. Since $q$ is extremal epimorphic and $i$ monomorphic, the left image $L$ of $f$ lies between, and there are 
connecting maps $h_{T} \rightarrow L \rightarrow G$, accomplishing the factorization of $\alpha$. Since $\gamma$ is extremal epimorphic, every map $\beta: Q(F) \rightarrow h_{Z}$ factors across the left image of $\beta \gamma$, which is $h_{Y}$ for some extremal quotient $Y$ of $\Sigma$. By 3.14, there are only a small set of these. $Q(F) \subset P$ is factored across their product, and an initial factor of an extremal monomorphism is an extremal monomorphism, completing the proof.

4.9. Every injective category is small-complete.

The proof is trivial except for one point that might be overlooked. Do not adjoin a limit or colimit functor, but the functor like $R_{D}^{0}$ in 2.1.

One would like to deduce 4.9 by embedding in a locally small smallcomplete extension. I do not know if it is possible. Of course there need not be such an extension in $\operatorname{Cat}\left(\mathfrak{C}^{*}, \mathfrak{U}\right)$. One could go step by step, adjoining every needed small limit or colimit, expecting to finish after $\infty$ suitably arranged steps; but while every small diagram will then have had a limit, the extensions are not conservative and there may be no limit at the end.

4.10. EXAMPLE. Not every ordinary category can be fully embedded in a complete locally small category.

The example is in $\$ 5$ and may, for all I know, be injective. There is another construction program that might lead to an embedding of any locally small category in an injective category, by adjoining one non-retractable object at a time. The adjoined objects can be chosen to be rather special grounding couples, and there is no conservation problem, but I do not see why the construction should ever ever end.

5. Examples. A complete category need not be injective, as was already suggested when we noticed wide-complete trivial categories. For trivial categories, injectiveness is equivalent to wide-completeness. It seems unlikely that every wide-complete locally small category is injective, but I have no example.

For 4.6 , consider the category $a$ faithfully grounded by $F: a \rightarrow \mathfrak{u}$, with $F(Q)$ consisting of $\infty$ one-point sets $X_{\alpha}$ and $\infty$ two-point sets $Z_{\beta}=\left\{0_{\beta}, 1_{\beta}\right\} ;$ the maps are the identities and one map $X_{\alpha} \rightarrow Z_{\beta}$ for each $\alpha$ and $\beta$, whose value in $\{0,1\}$ is a Kronecker delta $\delta_{\alpha \beta}$. The trivial category $\alpha$ has a trivial extension with one more object $Y$ across which all maps $X_{\alpha} \rightarrow Z_{\beta}$ factor. But $F$ cannot be extended; $F(Y)$ would have to include an image point of each $X_{\alpha}$, all distinct.

The incompletable example for 4.10 will be constructed in $u$ and small-complete, hence strict-complete; and the limitless multistrict analysis will be a part of the natural analysis of a map which does, as it happens, have a right image - for it is an extremal monomorphism, though not multistrict. For an example of a strict-complete category 
in which some map has no right image one would have to burrow deeper, and take care that the example is not (like this one) right wide-complete.

Consider first the trivial category of threads. For each isolated ordinal $\alpha>0$ there are a bare thread $T_{\alpha}$ and a capped thread $S_{\alpha}$. There is always a map $T_{\alpha} \rightarrow S_{\beta}$ and never a map $S_{\beta} \rightarrow T_{\alpha} ; T_{\alpha}$ maps to $T_{\beta}$ if and only if $\beta \geqq \alpha, S_{\alpha}$ to $S_{\beta}$ if and only if $\beta \leqq \alpha$. We adjoin a last point $P$, every thread mapping to $P$ and $P$ mapping to no thread, and ground the resulting category in disjoint sets as follows. $T_{\alpha}$ goes to a well-ordered set of order type $\alpha, S_{\alpha}$ to a well-ordered set of type $\alpha$ with an additional element, the $c a p c_{\alpha}$, and $P$ to a one-point set. No map except an identity has a cap as a value. Otherwise, all the maps between threads go by embedding as large an initial segment of the domain as the range will hold and mapping the rest of the domain, including cap, to the last element of the range. Now the union of all these sets is a set $U$ all of whose elements will be called points. $U$ is partially ordered if we say $p \leqq q$ when some map takes $p$ to $q$. If a least element were adjoined to $U$, the result would be a small-complete lattice, in which the successors of any point are totally ordered. Construct the set $V$ of marks from $U$ by replacing each two elements $u_{\alpha}, c_{\alpha}$, where $u_{\alpha}$ is the $\alpha$-th element of $S_{\alpha}$ and $c_{\alpha}$ the cap, by one element $v_{\alpha}$, a double mark. Maps of threads take marks into marks, and $V$ is another small-complete lattice-lacking-zero with totally ordered successors. The example $\mathfrak{C}$, the category of snarls, has for objects all small families of marks, i.e. functions $L \rightarrow V$, where $L$ is a small set of indices. Before defining snarl maps we ground $\lambda: L \rightarrow V$ to a set $F(\lambda)$ consisting of two elements for each indexed double mark and one element for each of the remaining indexed single marks. Then maps are functions $F(\lambda) \rightarrow F(\mu)$ which take each point to a successor and map each double mark either to one successor mark or to two last points (which may coincide).

One can readily check small-completeness of $\mathfrak{e}$. Products are Cartesian products of families, coproducts are disjointed unions, coequalizers are constructed by identifying and, where different marks are identified, taking suprema in $V$. The fact that that construction gives an epimorphism is plain from the consideration that the successors of a double mark are reverse well-ordered. For an equalizer, one takes a subfamily, except that the effect in a double mark $\left\{u_{\alpha}, c_{\alpha}\right\}$ of equalizing two maps which take it to last points and agree just on $u_{\alpha}$ is to replace this mark $v_{\alpha}$ by $v_{\alpha+1}$. (If the maps agree just on $c_{\alpha}$, this mark contributes nothing to the equalizer.)

The threads, being particular small families of marks, are fully embedded in the snarls. The capped threads $S_{\alpha}$ with their maps form 
a limitless multistrict analysis, the beginning of the natural analysis of any of the extremal monomorphisms $T_{\beta} \rightarrow S_{1}$. Moreover, if a limit $X$ were adjoined, it would have to have $\infty$ different maps to the snarl $Z$ consisting of two last points, for various left conical extensions by $T_{\beta}$ 's distinguish all formally different maps $X \rightarrow S_{\alpha} \rightarrow Z$ except those which differ only at the cap. 4.10 is proved.

5.1. EXAMPLE. A non-strict monomorphism as an initial factor of a strict monomorphism.

In the simplest example I know, two of the objects are accidentally isomorphic; the notation ignores this and follows 2.7. Let $X$ be the free semigroup on generators $x, y, z, X^{\prime}$ the quotient of $X$ by the congruence relation identifying $x z y$ with $z$. One can check, easily, that every word in $x, y, z$ is equal in $X^{\prime}$ to a unique word in which " $x z y$ " does not occur. Hence the subsemigroup $W$ of $X$ generated by $z, x z$, and $z y$ goes monomorphically into $X^{\prime}$. To verify that the monomorphism $W \rightarrow X^{\prime}$ is strict, one must examine the semigroup $Y$ presented by generators $x_{1}, x_{2}, y_{1}, y_{2}, z$ and relations $x_{1} z=x_{2} z, z y_{1}=z y_{2}, x_{1} z y_{1}=z$, to establish that every word there is equal to a unique equivalence class of words in which no string " $x_{i} z y_{i}$ " occurs and the equivalence consists of ignoring subscripts on $x$ 's preceding, or $y$ 's following, a $z$. Thus the two obvious embeddings of $X^{\prime}\left(x \rightarrow x_{i}, y \rightarrow y_{i}, z \rightarrow z\right)$ agree exactly on $W$. But the initial factor $W \subset X$ is not a strict monomorphism, $x z y$ being in the dominion; the example $W \subset X$ was given in [9] to show that the dominion can be a free extension.

5.2. Example. Retractions need not preserve limits.

Start with a disjoint sum of $\mathfrak{u}$ and the category $\mathfrak{u}_{0}$ of all small sets with base point. Adjoin a mapping $0: X \rightarrow Y$ for each $X$ in $\mathfrak{u}_{0}$, $Y$ in $\mathcal{u}$. The result is wide-complete, the full subcategory on all objects except the empty set $L$ of $\mathfrak{U}$ is still wide-complete, and there are retractions taking $L$ to any object $X$ of $\mathfrak{u}_{0} . L$ is an equalizer of maps between non-empty sets in $u$, and is not preserved unless it goes to the zero of $\mathfrak{u}_{0}$.

5.3. EXAMPLE. A full reflection of a right complete category that is not right complete.

First consider the category $\pi$ of all small sets having a marked point 0 and possibly two more marked points $p, q$, all three different. That is, there are two types of objects in $\Re$, those which lack both $p$ and $q$ and those which have both. The mappings are all functions $f$ satisfying $f(0)=0, f(p)=p, f(q)=q$, and $x \neq 0 \Rightarrow f(x) \neq 0$. This category would become right (wide-) complete if a right zero $R$ were adjoined. Then, coproducts involving $R$ would reduce to $R$, and other coproducts come from disjoint unions by identifying all 0 's, identifying 
all $p$ 's, identifying all $q$ 's. Coequalizers of pairs $f, g$ would be formed by making all identifications $f(x) \sim g(x)$ and collapsing to $R$ in case $p$ became identified to $q$. Since an epimorphism can omit at most two values, that would establish right completeness. But $R$ is missing from $\mathfrak{F}$. Let $L$ denote the left zero $\{0\}$.

Let $G$ be a totally ordered set consisting of the negative integers and the small ordinals in natural order. Let $\mathfrak{C}$ be a category with the following objects and maps. For each $\gamma \in G$, a copy $\Re_{\gamma}$ of $\Re$. One additional object, a left zero $L$ which is the range of no map except $1_{L}$. For $X_{\beta}$ in $\mathcal{F C}_{\beta}, Y_{\gamma}$ in $\mathfrak{F}_{\gamma}, \gamma>\beta$, there is one map from $X_{\beta}$ to $Y_{\gamma}$, none from $Y_{\gamma}$ to $X_{\beta}$. This defines $\mathcal{C}$, since compositions not within one $\mathfrak{T C}_{\gamma}$ have only one possible value.

$\mathcal{C}$ is right complete. For each $\mathcal{H C}_{\gamma}$, the missing coequalizer $R_{\gamma}$ is $L_{\gamma+1}$. Coproducts extending over many $\mathfrak{F}_{\gamma}$ are formed by ignoring all but the last $\mathfrak{K}_{\gamma}$; if there is no last, the coproduct is the next $L_{\delta}$. ( $L$ is the coproduct of the empty family.) As for coequalizers, distinct coterminal pairs of maps occur only within an $\mathfrak{H C}_{\gamma}$; strict epimorphisms can go no farther forward than from $\mathfrak{H C}_{\gamma}$ to $L_{\gamma+1}$. Since $L_{\gamma+1}$ has no strict quotient except itself, compositions of strict epimorphisms are strict, and we have right completeness.

Reflect $\mathfrak{C}$ upon a full subcategory by adjoining (in each $\mathfrak{F C}_{\gamma}$ ) the points $p, q$ to all objects lacking them; every map $f: X_{\gamma} \rightarrow Y_{\gamma}$ extends over $p$ and $q$ uniquely, and we have a reflector. The image $B$ is (by 3.1) right strict-complete. But in $B$ the strict epimorphisms $T_{\gamma}$ $\rightarrow L_{\gamma+1}(T=\{0, p, q\})$ are shifted to $T_{\gamma} \rightarrow T_{\gamma+1}$, and form a right multistrict analysis having no colimit.

I do not know whether the limits of multistrict analyses which arise in factoring a mapping are respected by retractions, or by adjoint retractions. (If $B$ is reflective full in $\mathcal{C}$ and $f: X \rightarrow Y$ in $B$ has a left image in $\mathbb{C}$ at the end of a multistrict analysis, reflection preserves the analysis but not the property of reaching all the way to the image. If the $B$-images of $f$ exist - the problem-they are the $\mathbb{C}$-images, since each essential property is preserved by one of the two adjoint functors.)

5.4. EXAmple. A full reflection of a right wide-complete category that is not right wide-complete.

For each small ordinal $\alpha>0$, let $\vartheta_{\alpha}$ be a copy of the category of all small non-empty sets. Let $\vartheta_{0}$ be a copy of all of $u$. Fill out the union by giving $\operatorname{Hom}(X, Y)$ one element for $X$ in $\vartheta_{\alpha}, Y$ in $\vartheta_{\beta}, \alpha<\beta$, and $\operatorname{Hom}(Y, X)$ no element. The resulting category $\mathfrak{C}$ is right smallcomplete by similar reasoning to that of 5.3 , and right wide-complete because there are no epimorphisms except within a $\mathcal{V}_{\alpha}$. But the full 
subcategory on the empty set from $\vartheta_{0}$ and the one-point sets from the other $\mathcal{V}_{\alpha}$ is plainly reflective and not right wide-complete.

5.5. ExAmple. An incomplete reflective subcategory of the abelian groups.

Let $D$ be the full subcategory of the category $\mathcal{G}$ of small abelian groups composed of the even-dimensional vector spaces over the integers mod 2. Of course $D$ is incomplete and not reflective in the standard embedding. But map $D$ into $\mathcal{G}$ by the abelian-principal functor $h^{\sharp}$, where $X$ is $Z_{2} \oplus Z_{2}$ and $h^{\sharp}(Y)$ is $h^{X}(Y)$ regarded as a group under pointwise addition. $h^{\#}$ is the restriction to $D$, in the standard

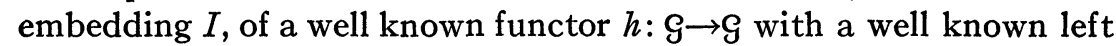
adjoint $\otimes X$. The image of $\otimes X$ is contained in $I(D)$, so that $\otimes X=I t$ for a tensor-functor $t: G \rightarrow D$. It is trivial to check that $t, h^{*}$ also are adjoint functors. ( $D(t(A), B)$ is $\mathrm{G}(I t(A), B), \mathrm{G}\left(A, h^{\#}(B)\right)$ is $\mathrm{g}(A, h(B))$.) As $h^{\#}$ is one-to-one on objects and mappings, its incomplete image is reflective.

\subsection{EXAMPLE. A left closed, full, non-reflective retract.}

This can be done in a trivial category constructed from the threads $T_{\alpha}, S_{\beta}$ of the category of snarls by adjoining an object $U$ with one map $T_{1} \rightarrow U$ and one map $U \rightarrow S_{\beta}$ for each $\beta$; the subcategory omitting $U$ is the indicated example.

5.7. EXAMPLE. A left adequate subcategory of a left adequate subcategory of a primitive category of algebras that is not coseparating.

The algebras have three unary operations, idempotent $\alpha$ and $\beta$ and an involution $\sigma$ satisfying $\alpha(\sigma(x))=\beta(x)$ (so $\beta \sigma=\alpha \sigma^{2}=\alpha$ ) and $\sigma \alpha=\alpha$, $\sigma \beta=\beta$. That is to say, it is the category $\mathfrak{C}$ of ordinary cogroundings of a one-object category $D$ whose object $D$ has the endomorphisms of a two-point set. $D$ and the one-point algebra $P$ form a subcategory $B$ of $\mathcal{C}$ that is left adequate in $\mathcal{C}$, since it contains $D$. $B$ is isomorphic with the full subcategory of $\mathcal{U}$ on a one- and a two-point set, and the full subcategory $a$ on $P$ is left adequate. But back in $\mathfrak{C}, D$ is free on one generator $x$, and has two embeddings $x \rightarrow y, x \rightarrow z$ in the algebra generated by $y$ and $z$ subject to $\alpha(y)=\alpha(z), \beta(y)=\beta(z)$, which are not coseparated by the trivial algebra $P$.

(One can replace $B$ by a complete subcategory $\mathcal{u}$. $Q$ is complete.)

5.8. Example. $A$ stable functor that is not a limit.

Of course, such a functor can be constructed almost at random, but then the proof will be complicated. All limits are apparent if we start with a free category generated by one map $\sigma: X \rightarrow X$. $X$ can be represented as a free algebra on one generator with one unary operation. Its limits are the empty algebra, the one-element algebra, and the powers of $X$; for the relations which a diagram of $X$ 's can impose on 
coordinates in the product can only be incompatible or make some determined by others. Thus the free algebra on two generators is not a limit of copies of $X$, though it is a retract of $X \times X$.

\section{REFERENCES}

1. B. Eckmann and P. Hilton, Group-like structures in general categories. II, Equalizers, limits, lengths, Math. Ann. 151 (1963), 150-186.

2. P. Freyd, Abelian categories, Harper and Row, New York, 1964.

3. H. Gaifman, Infinite Boolean polynomials, Fund. Math. 54 (1964), 229-250.

4. A. Grothendieck, Sur quelques points d'algèbre homologique, Tôhoku Math. J. (2) 9 (1957), 119-221.

5. A. Hales, On the non-existence of free complete Boolean algebras, Fund. Math. 54 (1964), 45-66.

6. J. Isbell, Adequate subcategories, Illinois J. Math. 4 (1960), 541-552.

7. - Two set-theoretical theorems in categories, Fund. Math. 53 (1963), 43-49.

8. - Subobjects, adequacy, completeness and categories of algebras, Rozprawy Mat. 36 (1964), 32 pp.

9. - Epimorphisms and dominions, Proc. Conference on Categorical Algebra (La Jolla, 1965) (to appear).

10. D. Kan, Adjoint functors, Trans. Amer. Math. Soc. 94 (1958), 294-329.

11. F. Lawvere, Functorial semantics of algebraic theories, thesis, Columbia University, 1963.

12. S. MacLane, Categorical algebra, Bull. Amer. Math. Soc. 71 (1965), 40-106.

13. J. Shepherdson, Inner models for set theory. III, J. Symbolic Logic 18 (1953), 145-167.

Case Institute of Technology 